\title{
Convergent and distributed effects of the $3 q 29$ deletion on the human neural transcriptome
}

\author{
Esra Sefik ${ }^{1,2,7}$, Ryan H. Purcell $\mathbb{D}^{3,4,7}$, The Emory 3q29 Project*, Elaine F. Walker ${ }^{2}$, Gary J. Bassell ${ }^{3,4}$ and Jennifer G. Mulle $\mathbb{D}^{1,5}$ 鴈
}

(c) The Author(s) 2021

The 3q29 deletion (3q29Del) confers high risk for schizophrenia and other neurodevelopmental and psychiatric disorders. However, no single gene in this interval is definitively associated with disease, prompting the hypothesis that neuropsychiatric sequelae emerge upon loss of multiple functionally-connected genes. 3q29 genes are unevenly annotated and the impact of 3q29Del on the human neural transcriptome is unknown. To systematically formulate unbiased hypotheses about molecular mechanisms linking 3q29Del to neuropsychiatric illness, we conducted a systems-level network analysis of the non-pathological adult human cortical transcriptome and generated evidence-based predictions that relate $3 q 29$ genes to novel functions and disease associations. The 21 protein-coding genes located in the interval segregated into seven clusters of highly co-expressed genes, demonstrating both convergent and distributed effects of 3q29Del across the interrogated transcriptomic landscape. Pathway analysis of these clusters indicated involvement in nervous-system functions, including synaptic signaling and organization, as well as core cellular functions, including transcriptional regulation, posttranslational modifications, chromatin remodeling, and mitochondrial metabolism. Top network-neighbors of 3q29 genes showed significant overlap with known schizophrenia, autism, and intellectual disability-risk genes, suggesting that 3q29Del biology is relevant to idiopathic disease. Leveraging "guilt by association", we propose nine 3q29 genes, including one hub gene, as prioritized drivers of neuropsychiatric risk. These results provide testable hypotheses for experimental analysis on causal drivers and mechanisms of the largest known genetic risk factor for schizophrenia and highlight the study of normal function in non-pathological postmortem tissue to further our understanding of psychiatric genetics, especially for rare syndromes like 3q29Del, where access to neural tissue from carriers is unavailable or limited.

Translational Psychiatry (2021)11:357; https://doi.org/10.1038/s41398-021-01435-2

\section{INTRODUCTION}

Copy number variants (CNVs) offer tractable entry points to investigate the genetic contributions to complex neuropsychiatric diseases. The recurrent $1.6 \mathrm{Mb}$ deletion of the $3 q 29$ interval (3q29Del) is robustly associated with schizophrenia spectrum and other non-affective psychotic disorders (SZ) [1-4] and is the strongest known risk allele for the disease with an estimated odds ratio $>40$ [5]. The associated syndrome is a rare $(\sim 1$ in 30,000$)$ and typically de novo genomic disorder that is often accompanied by reduced birth weight, failure to thrive, dysmorphic craniofacial features, and varied medical manifestations, including congenital heart defects [6-8]. Autism spectrum disorders (ASD) and intellectual disability/developmental delay (IDD) are also enriched in 3q29Del carriers $[7,9,10$,$] . However, it is not currently known which genes$ within the interval are responsible for the increased neuropsychiatric risk. No single 3q29 interval gene has been definitively associated with SZ, ASD, or IDD, prompting the hypothesis that haploinsufficiency of more than one gene is required [11]. The paucity of information regarding the functional roles of most 3q29 interval genes hampers the development of evidence-based hypotheses for deciphering this link. No transcriptomic investigation of 3q29Del in humans has been reported, and it is unclear what impact hemizygous loss of these genes might have in the nervous system.

Among the 21 protein-coding genes of the 3q29Del locus, several have been proposed as drivers of the behavioral phenotypes [12], yet the evidence for their individual association with neuropsychiatric disease remains suggestive. DLG1 produces a synaptic scaffold protein that interacts with AMPA and NMDAtype glutamate receptors [13-16], the latter of which is hypothesized to be involved in SZ pathogenesis [17]. A DLG1 polymorphism has been genetically linked to SZ [18,19,]. However, the mouse-specific phenotypes of 3q29Del are not recapitulated by haploinsufficiency of Dlg1 alone [20]. Another prominent candidate, PAK2 encodes a brain-expressed protein kinase involved in cytoskeletal dynamics [21] and dendritic spine morphology [22]. Both DLG1 and PAK2 are homologous to genes linked to IDD $[23,24$,$] and evidence from Drosophila indicates that$ joint haploinsufficiency of both genes simultaneously may be required for synaptic defects rather than either gene individually [25]. A recent study generated select combinatorial knockdowns of 3q29 gene homologs in Drosophila and Xenopus laevis and proposed that a component of the nuclear cap-binding complex,

\footnotetext{
${ }^{1}$ Department of Human Genetics, Emory University School of Medicine, Atlanta, GA, USA. ${ }^{2}$ Department of Psychology, Emory University, Atlanta, GA, USA. ${ }^{3}$ Department of Cell Biology, Emory University School of Medicine, Atlanta, GA, USA. ${ }^{4}$ Laboratory of Translational Cell Biology, Emory University School of Medicine, Atlanta, GA, USA. ${ }^{5}$ Department of Epidemiology, Rollins School of Public Health, Emory University, Atlanta, GA, USA. ${ }^{7}$ These authors contributed equally: Esra Sefik, Ryan H. Purcell. *A full list of author affiliations appears at the end of the paper. ${ }^{\bowtie}$ email: jmulle@emory.edu
}

Received: 7 October 2020 Revised: 29 April 2021 Accepted: 7 May 2021

Published online: 15 June 2021 
NCBP2 [26], mediates neurodevelopmental defects in 3q29Del syndrome [11]. However, in the 3q29Del mouse model, Ncbp2 is not decreased at the protein level in brain tissue [20], dampening enthusiasm for this gene as a causal element. It remains unclear which genes and their potential interactions are responsible for 3q29-associated phenotypes.

To avoid annotation bias [27] and address the knowledge gap for 3 q29 genes, we employed a gene co-expression network analysis approach that is rooted in systems biology [28-31], and generated evidence-based predictions that relate individual 3q29 genes to novel functions and disease phenotypes. Accumulating evidence indicates that genes work in conjunction, rather than in isolation, to realize most cellular functions [11,25,32,]. Genes participating in the same molecular and biological pathways tend to show positively correlated expression with each other (coexpression), as they are often expressed under the control of a coordinated transcriptional regulatory system [33,34,]. In this holistic context, well-characterized genes can be leveraged to infer the functions of understudied genes by studying network patterns that emerge by means of co-expression [35-37]. This in silico approach to investigating unknown biology extends the "guilt by association" paradigm [38] that is extensively used for inductive reasoning in other domains to gene-gene interactions in complex biological systems [39-41]. Weighted gene co-expression network analysis (WGCNA) [29,42,] has been successfully deployed to study how genes embedded in network structures jointly affect complex human diseases [43-53]. We employed this paradigm to glean new biological insights into the 3q29Del syndrome.

\section{METHODS AND MATERIALS \\ The reference dataset}

To uncover the network-level operations of genes located in the recurrent 3q29Del locus, we employed WGCNA [29,42,] and organized the nonpathological adult human cortical transcriptome into modules of highly coexpressed genes (Fig. 1a). Given the strong genetic link between 3q29Del and risk for SZ [5], we focused the present network analysis on revealing the clustering patterns of 3q29 interval genes as a function of their expression similarity during adulthood: a period when SZ typically manifests diagnostically, with peak onset in late adolescence and early adulthood [54], and a substantial proportion of patients becoming ill during middle adulthood [55]. A prior study has shown that only $0.7 \%$ of genes detected in the neocortex show a temporally regulated profile of differential expression during adulthood (between ages 20-60 years); [56] hence, gene expression data were pooled across adulthood to derive the present dataset. We further focused our analysis spatially on gene-gene relationships in the prefrontal cortex (PFC): a brain region that subserves a diverse range of cognitive and emotional operations, is implicated in the etiology of SZ and may be particularly vulnerable to the effects of genetic disruption due to its protracted development [57]. For these reasons, the network was constructed on publicly available transcriptomic data from the Genotype Tissue Expression Project (GTEx) [58], using PFC (Brodmann Area 9) samples from male and female adults (age range $=20-79,68.2 \%$ male) with no known history of psychiatric or neurological disorder (Fig. S1 and Table S1.1). Transcriptome profiling was performed by RNA-Seq as described in [58].

Protein-coding transcripts were extracted from the dataset, normalized expression values were $\log _{2}$ transformed and summarized at the genelevel, and outlier samples were removed (Fig. S2). The data were corrected for covariance mediated by age, sex, death classification, postmortem interval (PMI) and batch effect [59,60,] (Fig. 1b and Fig. S1). Genes with zero variance and genes and samples with greater than $50 \%$ missing entries (default) were removed $[29,42$,$] . The normalized, outlier-removed, residua-$ lized expression values of 18,410 protein-coding genes from 107 samples constituted the final dataset.

\section{Weighted and signed gene co-expression network construction}

The single-block pipeline implemented in the WGCNA R package was employed for network construction [29,42,]. Co-expression similarity was defined by biweight midcorrelation $[61,62$,$] . To capture the continuous$ nature of interactions and accentuate strong positive correlations, co- expression similarity was transformed into a signed and weighted adjacency matrix by a soft-thresholding procedure that yielded approximate scale-free topology [63-66] (Fig. 1c and Fig. S3). Topological overlap measures (TOM) were calculated from the resulting adjacency matrix to capture not only the univariate correlational relationship between gene pairs but also the large-scale connections among "neighborhoods" of genes [67,68,] (Fig. 1a). Hence, we measure the interconnectedness of gene pairs in relation to the commonality of the nodes they connect to.

The modular structure of the data was revealed by average linkage hierarchical clustering on TOM following its transformation into a dissimilarity metric (Fig. 1d). Module definitions used in this study do not use a priori knowledge of functionally defined gene sets. Instead, modules were detected in a data-driven fashion through adaptively pruning the branches of the resulting dendrogram by the dynamic hybrid tree-cut method [69]. The expression profile of each identified module was subsequently summarized by a module eigengene (ME) [70], defined as its first principal component. Calculation of MEs amounted to a data reduction method used for effectively correlating entire modules to one another and for establishing the eigengene-based module connectivity measure (kME) of individual genes [42]. To eliminate spurious assignment of genes into separate modules, modules with strongly correlated MEs were amalgamated (Pearson's $r>0.8, P<0.05$, cut height $=0.2$ ) (Fig. 1d).

\section{Module preservation and quality analyses}

To validate the reproducibility of the network modules derived from the GTEx dataset (considered the reference dataset/network), we evaluated module preservation in an independently-ascertained, demographicallycomparable transcriptomic dataset, referred to as the test dataset/network (Fig. S4a). For this purpose, publicly available transcriptomic data was obtained from the BrainSpan Developmental Transcriptome Project [56]. Thirty non-pathological postmortem samples from the PFC of male and female adults (age range $=18-37,50 \%$ male) with no known neurological or psychiatric disorder comprised the test dataset (Fig. S4b and S1.1). Transcriptome profiling was performed by RNA-Seq as described in [71].

The preprocessed test dataset consisted of normalized and residualized expression values for 18,339 protein-coding genes from 30 samples that were pooled from four subregions of the PFC to test whether the co-expression patterns derived from Brodmann Area 9 of the PFC in the reference dataset could be commonly found and robustly defined in broader sampling of tissue across the PFC. Prior to preservation analysis, a sample-level hierarchical clustering of the test dataset was conducted, which revealed no distribution bias associated with PFC sub-region, ruling out tissue of origin as a potential confound in test network construction. (Fig. S4c).

To determine whether properties of within-module topology were preserved in the test network, we calculated a composite, network-based preservation statistic for each module $\left(Z_{\text {summary.pres }}\right)$ by using the modulePreservation function of the WGCNA package in $R$ [72]. $Z_{\text {summary }}$. pres is a summary statistic that encompasses multiple density-based and connectivity-based preservation statistics, which are equally important for establishing the overall preservation of a module. To determine whether the observed preservation statistics were higher than expected by chance, we randomly permuted the module assignments in the test dataset (200 times) and derived a standardized $Z_{\text {summary.pres }}$ score for each module. To account for this metric's dependence on module-size, we reduced large modules by randomly sampling 1000 intra-modular nodes. The resulting scores were evaluated according to established thresholds: $Z_{\text {summary.pres }}<$ 2 , no evidence for preservation; $2<Z_{\text {summary.pres }}<10$, moderate evidence for preservation; $Z_{\text {summary.pres }}>10$, strong evidence for preservation.

In addition to testing preservation, we measured the quality of the modules that were defined in the reference network by employing a resampling technique that applied the preservation statistics outlined above to repeated random splits of the reference dataset. We assessed the robustness of the identified modules (i.e., how distinct a module is from the background) by calculating a standardized, composite quality statistic $\left(Z_{\text {summary.qual }}\right)$, as described in [72]. The same $Z_{\text {summary.pres }}$ thresholds outlined above were used to evaluate $Z_{\text {summary.qual }}$. The code written for WGCNA implementation in the current paper is available from authors upon request.

\section{Functional characterization of network modules harboring $3 q 29$ genes}

Pathway analyses of individual modules found to harbor $3 q 29$ genes were performed by g:Profiler (http://biit.cs.ut.ee/gprofiler), using annotations from the Gene Ontology (GO), Reactome, and Kyoto Encyclopedia of Genes 
a

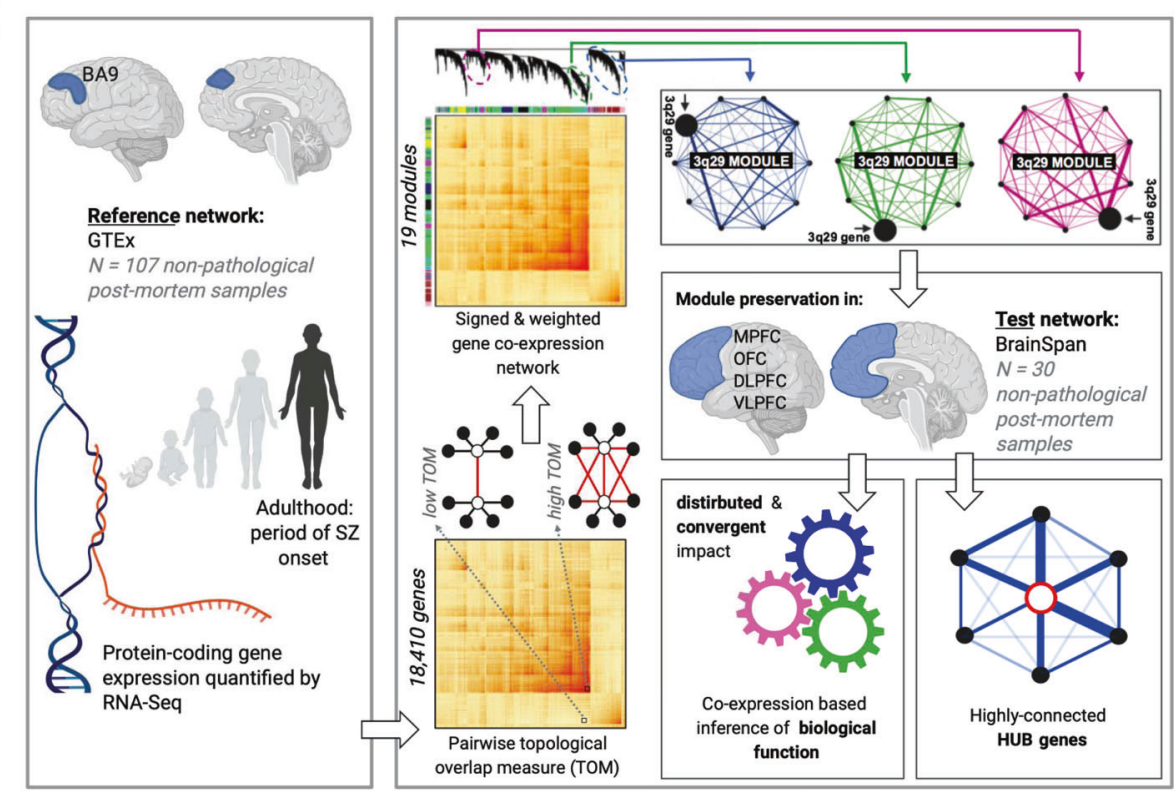

b

C
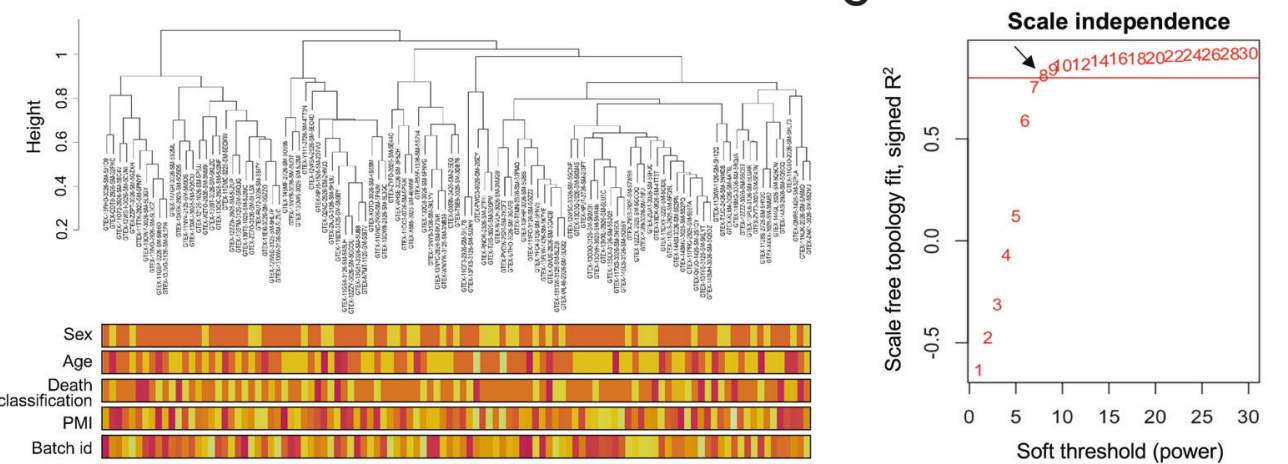

d
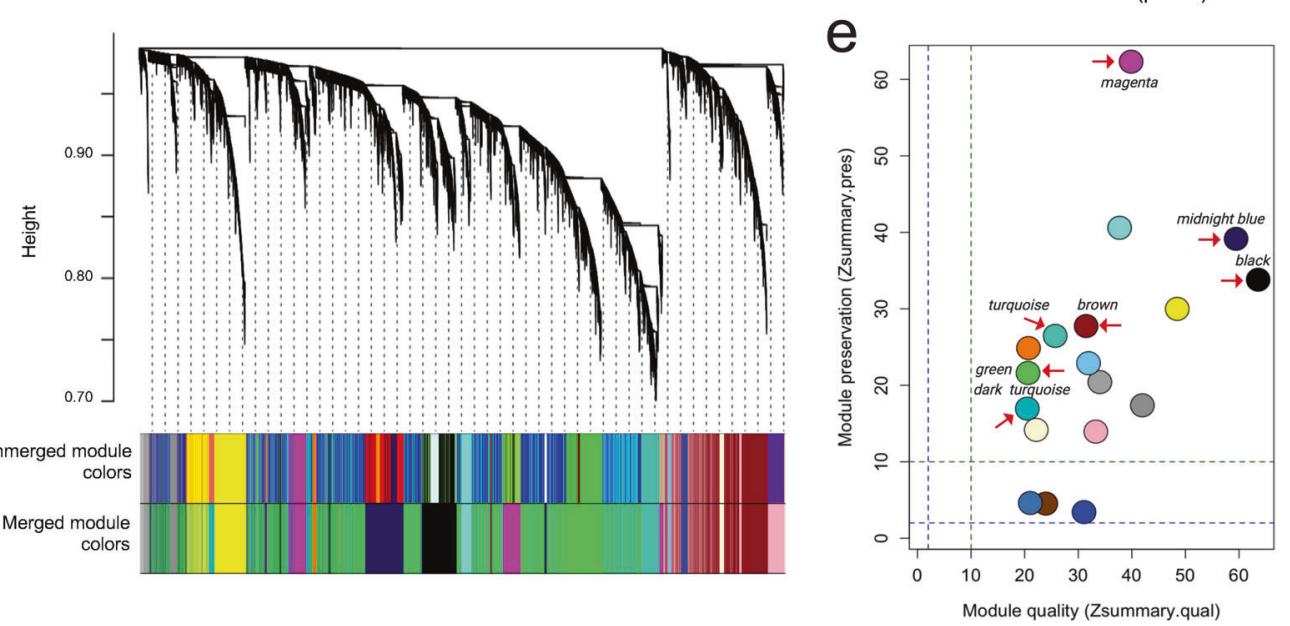

and Genomes (KEGG) databases. Enriched terms surpassing the adjusted g: SCS significance threshold of $P<0.05$ were filtered for size and semantic similarity [73].

To further interrogate whether the gene co-expression modules identified in this study represent biologically meaningful units with shared membership of the same molecular complex or functional pathway, we also investigated whether the genes co-clustering in the same transcriptomic module tend to interact at the protein level. First, we queried the known protein interactors of $3 q 29$ interval genes based on the Human Reference Protein Interactome Mapping Project (HuRl; http:/interactome-atlas.org/) (see [74]). We identified qualitative overlaps between these known protein interactors and gene co-expression partners of 3q29 interval genes at module and meta-module levels of network organization. Second, we tested the co-expression modules harboring 3q29 interval genes for enrichment of known and predicted protein-protein interactions (PPIs) curated from the STRING database (v.11, https://string-db.org/). The STRING enrichment analysis tool was used to test whether the observed number of protein interactions (edges) in each interrogated module is significantly higher than the number of edges expected if the nodes were to be selected from the genome at random (see [75]). See Supplemental Methods for details. 
Fig. 1 Unbiased weighted gene co-expression network analysis (WGCNA) of the human transcriptome in the healthy adult prefrontal cortex (PFC). a A schematic of the data analysis workflow underlying WGCNA-derived predictions for functional interrogation of the 3q29Del interval. The reference dataset was obtained from the GTEx Project to construct a systems-level network representation of coordinated gene expression patterns across 107 non-pathological postmortem samples collected from the Brodmann Area 9 (BA9) of male and female adults with no known history of psychiatric or neurological disorder. Modules of highly co-expressed genes were identified based on their topological overlap measure (TOM). The TOM between two genes is high if the genes have many overlapping network connections, yielding an interconnectedness measure that is proportional to the number of shared neighbors between pairs of genes. The resulting network was screened for modules harboring 3q29 interval genes (3q29 modules), which were then interrogated for biological function and hub genes. A test dataset obtained from the BrainSpan Project was used to validate the reproducibility of this network in an independent sample of 30 nonpathological postmortem specimens collected from four subregions of the PFC from adult males and females with no known history of psychiatric or neurological disorder. These subregions are the OFC orbital frontal cortex, DLPFC dorsolateral PFC, VLPFC ventrolateral PFC, and MPFC medial PFC. b Sample-level dendrogram and trait heatmaps of the reference dataset. The dendrogram was yielded by hierarchical clustering of 107 GTEx samples using normalized, outlier-removed, and residualized gene expression values for 18,410 protein-coding genes. Color bars represent trait heatmaps for sex, age-group (range $=20-79$ years), death-classification based on the Hardy scale $($ range $=0-4$ ), postmortem interval (PMI), and batch id. The color intensity (from light yellow to red) is proportional to continuous or categorical values (in increasing order) of each variable. For sex, yellow and orange indicate female and male, respectively. Transcriptomic data were corrected for covariance mediated by these variables prior to network construction. Adjusted data reveal no distribution bias associated with the interrogated confounds in sample-level clustering patterns. c Determination of the soft-thresholding power $(\beta)$ used for WGCNA. A $\beta$ of 8 (black arrow) was identified as the lowest possible power yielding a degree distribution that results in approximate scale-free network topology (SFT $R^{2}$ fit index $=0.8$; red line). d Clustering dendrogram and module assignments of genes, with dissimilarity based on TOM. 18,410 protein coding genes (leaves = genes) clustered into 19 final modules (bottom color bar), detected by the dynamic hybrid tree cut method. Modules with strongly correlated eigengenes (Pearson's $r>0.8, P<0.05$ ) were amalgamated to eliminate spurious assignment of highly coexpressed genes into separate modules. Color bars reflect module assignments before and after the merging of close modules. e Composite Zsummary scores for module-preservation (how well-defined modules are in an independent test dataset) and module-quality (how welldefined modules are in repeated random splits of the reference dataset). Permutation tests were performed to adjust the observed preservation and quality statistics of each module for random chance by defining Z statistics. All modules (labeled by color) identified in the reference network were preserved (reproducible) in the test network (Zsummary > 2; blue line). Overall, 15 out of 18 modules, including all 3 q29 modules (red arrows), exhibited strong preservation (Zsummary > 10; green line). 3/18 modules exhibited moderate preservation ( $2<$ Zsummary $<10$ ). All modules demonstrated strong evidence for high quality (Zsummary $>10)$, confirming that the modules identified in the reference network were well-defined and nonrandom.

\section{Identification of prioritized driver genes and biological mechanisms}

Disease-associated genes are often more closely connected to each other than random gene pairs in a biological network; this nonrandom network characteristic has enabled the identification of novel genetic risk loci for many diseases [76-80]. To generate data-driven hypotheses about which $3 q 29$ genes are causally linked to the major neuropsychiatric phenotypes of 3q29Del, we tested the overlap between "top neighbors" of individual 3 q29 genes and known risk genes for SZ and related disorders. A top neighbor was defined as any node whose gene expression profile has a moderate-to-high correlation (Spearman's rho $(\rho) \geq 0.5), P<0.05$ ) with a given 3q29 gene (considered a "seed") within the same module. Hence, top neighbors were identified by a hard-thresholding method applied only to intramodular edges of a seed that were initially defined by the topological overlap principle. Hypergeometric tests were conducted to gauge the probability of the overlap between curated gene sets and top neighbors, as implemented in the GeneOverlap package in R [81], followed by Benjamini-Hochberg multiple testing correction.

Lastly, to formulate testable hypotheses about key biological mechanisms that link the $3 q 29$ locus to neuropsychiatric disease, we interrogated the functional enrichment of prioritized driver genes, using the same pathway analysis approach applied to modules. See Supplemental Methods for details.

\section{Proof of concept for testing the validity of WGCNA-based predictions}

A necessary step in determining the utility of network-based predictions is a proof of concept of their validity in an experimental system. To this end, we assessed the validity of our WGCNA-derived predictions by testing the enrichment of co-expression network-partners of $3 q 29$ interval genes for differential expression in a mouse model of 3q29Del [20].

Mice harboring a heterozygous deletion of $1.26 \mathrm{Mb}$ (Del16 $\left.{ }^{+/ \mathrm{Bdh} 1-\mathrm{Tfr}}\right)$ that is homologous to the human 3q29Del locus were generated by CRISPR/ Cas9 technology previously [20]. At postnatal day seven, five mutant and five wild-type (WT) male pups were anesthetized under isoflurane and rapidly decapitated. The bilateral cortical sheet was dissected, chopped with a scalpel, and homogenized in QIAzol (Qiagen) in a Bullet Blender Tissue Homogenizer (Next Advance, Inc., Troy, NY). Total RNA was isolated using the miRNeasy Mini Kit (Qiagen) with on-column DNAse I treatment (Qiagen). RNA-sequencing libraries were generated using the SMART-Seq Stranded Kit (Takara Bio, Mountain View, CA). 50 M paired-end 150 bp read sequencing was performed on an Illumina platform. Sequences were quality-checked and aligned to the $\mathrm{mm} 10$ reference genome. Gene quantification was conducted using HTSeq-count [82]. We used two analysis tools (DESeq2 [83] and edgeR [84]) to identify differentially expressed genes (DEGs). Only the protein-coding consensus DEGs with nominal significance $(P<0.05)$ were carried into downstream analysis.

The statistical significance of the overlap between identified DEGs and the network co-expression partners of 3q29 interval genes was tested via hypergeometric tests, using the GeneOverlap package in $R$ [81]. All compared gene sets were filtered for mouse-human homology based on the HomoloGene database of the National Center for Biotechnology Information (NCBI) [85]. All procedures were performed under guidelines approved by the Emory University IACUC. See Supplemental Methods for details.

\section{RESULTS \\ Unbiased gene co-expression network analysis reveals convergent and distributed effects of 3q29 interval genes across the adult human cortical transcriptome}

Applying an unsupervised WGCNA approach [29,42,] to publicly available data from the GTEx Project [58] revealed that the protein-coding transcriptome of the healthy adult human PFC can be organized into a gene co-expression network of 19 modules (labeled by color) (Fig. 1d and Table S1.2). The identified modules group genes with highly similar expression profiles and likely represent shared function and/or co-regulation. The resulting module sizes ranged from 43 (steel-blue module) to 4746 (green module) genes, with an average module size of 1014 genes. To obtain high-quality module definitions, one module (gray module) was reserved for genes that could not be unequivocally assigned to any module. Thus, the gray module was excluded from downstream analysis. The resulting set of modules was screened for membership of 3q29 genes; modules that were found to harbor at least one 3q29 gene are referred to as "3q29 modules". Refer to Table S1.3 for gene ids and full names of 3q29 interval genes.

To ensure the reproducibility and robustness of our network analysis results, we tested the preservation and quality of the 
identified modules in an independent dataset obtained from the BrainSpan Developmental Transcriptome Project [56] (Fig. S4) and in repeated random splits of the reference dataset. All identified modules, except for the gray module (unassigned genes), were found to be successfully preserved in the test network $\left(Z_{\text {summary }}\right.$ pres $>2$ ) (Fig. 1e, Fig. S5 and Table S1.4). Specifically, 3/18 modules exhibited moderate evidence of preservation $\left(2<Z_{\text {summary.pres }}<\right.$ 10 ), and 15/18 modules, including all 3q29 modules, exhibited strong evidence of preservation $\left(Z_{\text {summary.pres }}>10\right)$. In addition to preservation statistics, we calculated multiple module quality statistics that measure how well-defined or robust the boundaries of individual modules are in the reference network. All 18 modules showed strong evidence for high cluster quality $\left(Z_{\text {summary.qual }}>\right.$ 10), revealing robust module definitions (Fig. 1e, Fig. S5, and Table S1.4). Specifically, all 3q29 modules had a $Z_{\text {summary.qual }}$ score $\geq 20$. These analyses revealed the replicable, well-defined, and nonrandom nature of the identified network modules. For extended results, see Supplemental Results.

The 21 protein-coding genes located in the $3 q 29$ interval clustered into seven modules (Fig. 2a-c): black (one 3q29 gene), brown (four 3q29 genes), dark-turquoise (one $3 q 29$ gene), green (six 3q29 genes), magenta (one 3q29 gene), midnight-blue (three $3 q 29$ genes), and turquoise (five 3 q29 genes). Within this network, $18(86 \%)$ of the $3 q 29$ interval genes concentrate into just four modules (Fig. 2a), suggesting that the haploinsufficiency of sets of genes within the locus may perturb the same biological processes via multiple hits, cumulatively disrupting redundancy and compensatory resiliency in the normative regulation of cellular functions.

To evaluate whether modules further clustered within larger meta-modules that represent the higher-order organization of the transcriptome, we identified meta-modules as tight clusters of positively correlated MEs, detectable as major branches of the eigengene dendrogram [86] (Fig. 2a, b). Meta-modules were screened to identify the grouping patterns among 3q29 modules, allowing exploration of extra-modular interactions. This analysis revealed that the 3q29 modules further cluster into three higher level meta-modules (Fig. 2b), which likely reflect dependencies and interactions between pathways involving $3 q 29$ genes. Simultaneously, leading presumptive candidates DLG1 and PAK2 (Fig. S6) were found in opposite branches of the network, demonstrating the distributed effects of this CNV across the transcriptomic landscape.

\section{Pathway analysis points to functional involvement of the $3 q 29$ locus in nervous-system functions and core aspects of cell biology}

Since highly co-expressed genes often share similar functions $[33,34$,$] , biological processes and pathways that are enriched in a$ co-expression module can be used to infer functional information for poorly annotated genes of that module. Functional enrichment analysis of 3q29 modules showed that their constituent genes converge onto canonical biological processes and known/ predicted PPI networks at proportions greater than expected by chance, indicating that these modules are biologically relevant units (Fig. 2d, Fig. S7-S9, and Tables S1.5-S1.8).

The turquoise and green modules showed overrepresentation of roles specific to the neuronal system and implicate involvement in multiple synaptic properties. Other 3q29 modules point to biological pathways that may also underlie neuropsychiatric pathology in 3q29Del. The magenta module was predominantly enriched for protein modification, turnover, and localization. Additionally, a link was identified between the magenta module and the initiation of major histocompatibility complex class-I (MHC-I)-dependent immune responses, driven by a genomic locus implicated in the etiology of SZ $[87,88$,$] . On the other hand,$ overrepresented pathways in the black module encompass regulation of gene expression and maintenance of the integrity of the cellular genome, including DNA repair, and the metabolism and processing of RNA. The midnight-blue module shared enriched roles with the black module, validating their shared meta-module structure, yet it was set apart by its involvement in cell cycle regulation. The brown module revealed primary enrichment for cellular metabolism and mitochondrial function, whereas the dark-turquoise module coalesced genes involved in epigenetic mechanisms and in signal transduction pathways mediated by Rho GTPases. This latter function may be attributable to PAK2, which encodes a known Rho GTPase effector. Intriguingly, this module was also enriched for estrogen receptor-mediated signaling, suggesting a potential mechanism for sex-specific effects. Taken together, functional characterization of the 3q29 modules point to novel mechanisms of shared or synchronized action for co-clustering 3q29 interval genes (Fig. 2d and Fig. S9).

Simultaneously, PPI network enrichment analysis revealed that all 3q29 modules show significant enrichment for PPIs that were systematically curated from the STRING protein interactome database (Fig. S8 and Table S1.8), augmenting confidence in our RNA-Seq based network predictions with proteomic evidence (midnight blue, black, brown, and magenta modules: $P$ value $<1.00 \mathrm{e}-16$; dark turquoise module: $P$ value $=1.11 \mathrm{e}-16$; green module: $P$ value $=8.62 \mathrm{e}-08$; turquoise module: $P$ value $=4.30 \mathrm{e}-09$ )

Additionally, we identified qualitative overlaps between the transcriptomic co-expression partners of 3q29 interval genes identified via WGCNA and known protein partners of 3q29 interval genes curated from the HuRI database (Fig. S7 and Table S1.7). Notably, of the 21 protein coding genes located in the $3 q 29$ interval, only 14 were found to have an entry on HuRI, $50 \%$ of which had less than eight known proteome-wide interactors. For reference, in the yeast proteome, an average of five interactors are estimated per protein [89]. Given that the average domain content of human proteins is higher than that of yeast [90], a much higher number of PPIs per protein is expected in humans. This finding is consistent with prior studies reporting that technological limitations in measuring the proteome with enough coverage results in a high rate of missing entries, which leads to significant bias and loss of information on human PPIs that may be disease relevant [91-93]. The missing PPI data for over one fourth of the genes located in the 3q29 interval corroborate the paucity of information regarding the functional roles of most 3q29 interval genes, and further reveal the need for novel approaches that are free of annotation bias [27]. The full list of PPIs curated from HuRI and STRING, and brief statistics and visual illustrations of the resulting PPI networks can be found in Fig. S7-S8 and Tables S1.7-S1.8. For extended results, see Supplemental Results.

UBXN7 is a highly connected cortical hub-gene predicted to play a crucial role in the neuropsychiatric sequela of 3q29Del Targeted disruption of a highly-connected "hub" gene produces a more deleterious effect on network function and yields a larger number of phenotypic outcomes than randomly selected or less connected genes [94,95,]. Hence, we sought to measure how strongly connected individual $3 q 29$ genes are to their modules by evaluating their intramodular kME (Fig. $2 \mathrm{c}$ and Table S1.3), defined as the Pearson's correlation between the expression profile of a gene and the eigengene of its assigned module [29,42,70,].

Genes with high intramodular kME are considered hub genes that are predicted to be critical components of the overall function of their module [29]. Nodes with high intramodular kME often have high intramodular connectivity (kIM), which reflects sum of adjacencies to other nodes [42]. However, an advantage of using kME over other connectivity metrics, is its defined $P$ value and values that lie between -1 and 1 , allowing comparison across modules that differ in size. To generate rigorous predictions about which 3q29 genes, if any, are intramodular hub genes, we adopted a conservative criterion that defines hub genes as nodes 


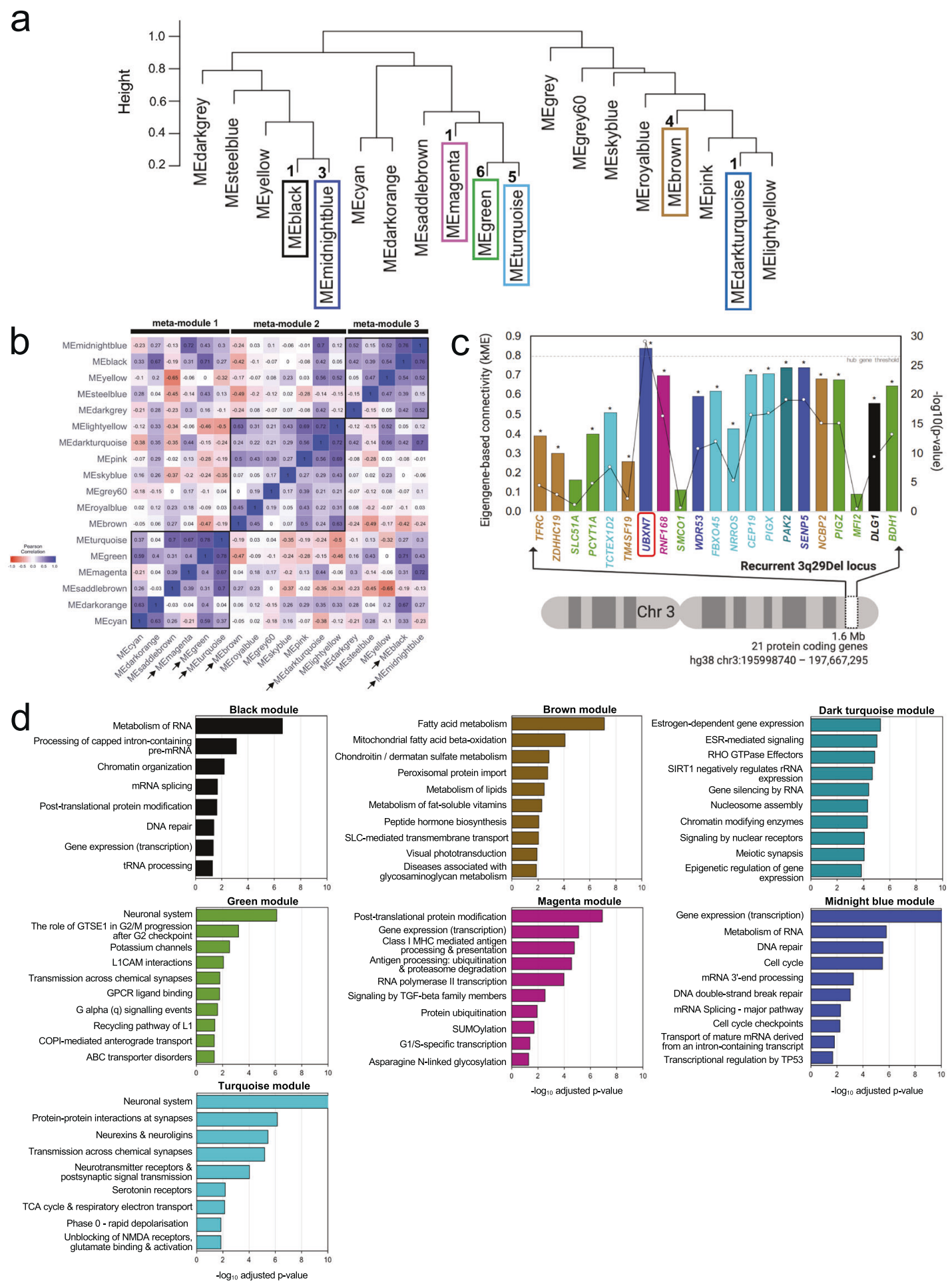

with $\mathrm{kME}>0.8 \quad(P<0.05)$. Only one $3 q 29$ interval gene was identified as a hub gene: UBXN7 (kME $=0.84, P=8.33 \mathrm{E}-30)$, which encodes a ubiquitin ligase-substrate adapter [96,97,].

SMCO1, SLC51A, and MFI2 had non-significant kMEs $(P<0.05)$ for their module, suggesting low kIM. These $3 q 29$ genes are detected but display very low abundance in the human cerebral cortex [98] (Table S1.6), which may relate to their peripheral network assignments in our analysis. Consequently, SMCO1, SLC51A, and $M F I 2$ were excluded from downstream analysis to derive the most parsimonious prioritization of driver genes based on tight network connections. A complete list of gene sets for each module and kME values are provided in Tables S1.2-S1.3.

Nine 3q29 interval genes form transcriptomic subnetworks enriched for known SZ, ASD, and IDD-risk genes

We next identified a refined subset of target genes (top neighbors) that not only co-cluster based on TOM but also have a strong pairwise correlation with 3q29 genes (Fig. 3a). Several 3 q29 genes were found to be top neighbors of one another. FBXO45 $(\rho=0.5, P=5.43 \mathrm{E}-09)$ and PIGX $(\rho=0.6, P=1.24 \mathrm{E}-10)$ are 
Fig. 2 Network-based inference of the functional impact of 3q29Del on the adult human prefrontal cortex (PFC). a Hierarchical clustering of module eigengenes (ME) that summarize the 19 modules identified by WGCNA. The 21 protein-coding genes located in the recurrent 3q29Del locus were found to be distributed into seven co-expression modules (3q29 modules; framed), The numbers next to dendrogram branches indicate the total number of 3q29 interval genes found in each 3q29 module. b Heatmap representing the strength of Pearson's correlation ( $r$ ) between ME-pairs. The seven 3q29 modules (arrows) further clustered into three higher-level meta-modules, corresponding to squares of blue color (high positive correlation) along the diagonal, also detected as major dendrogram branches in (a). c Eigengene-based connectivity strength (kME; $y$-axis) of 3q29 interval genes (x-axis; in chromosomal order) within their respective modules. kME is defined as the Pearson's correlation between a query gene and a given ME. The line graph indicates the $-\log _{10}(P$ value) of the plotted correlation coefficients ( $z$-axis); the asterisks above the graph indicate $P<0.05$. kME $>0.8(P<0.05$; dotted line) indicates hub (highly connected) gene status. UBXN7 (red frame) was found to be the only hub gene (kME $>0.8, P=8.33 \mathrm{E}-30)$ within the 3q29Del locus. SMCO1 $(\mathrm{kME}=0.11, P=0.25), S L C 51 A(\mathrm{kME}=0.17, P=$ 0.09 ), and $M F / 2$ ( $k M E=0.09, P=0.35)$ were found to have non-significant kMEs $(P>0.05)$ for their respective modules, suggesting peripheral membership. Color indicates module label. d Top ten biological pathways (Reactome database) significantly enriched in $3 q 29$ modules (adjusted- $P<0.05$; capped at $-\log _{10}$ (adjusted- $P=10$ )). The g:SCS method was used for multiple testing correction. The observed enrichment profile of the queried modules for known biological processes and pathways indicates that genes co-clustering in $3 q 29$ modules show coordinated expression and converge upon overlapping biological functions, more than expected by chance. The functional associations of gene sets comprising individual 3q29 modules were leveraged to infer likely molecular consequences of 3q29Del in the adult human PFC.

top-neighbors of CEP19, while SENP5 and WDR53 are topneighbors of each other $(\rho=0.5, P=1.05 \mathrm{E}-07)$ (Table S2.1). On the other hand, intramodular connections of TM4SF19 and ZDHHC19 did not meet top neighborhood criteria; hence they were not included in downstream analysis. Similar to SMCO1, $S L C 51 A$, and MFI2, their mRNA expression profiles indicate low abundance in the cerebral cortex (Table S1.6), which likely reflects their lack of strong network connections.

The human transcriptome is theorized to demonstrate nonrandom topological characteristics, where disease genes interact with other disease genes that underlie a common pathophenotype [99]. Concordant with this prediction, within the top neighbors of $3 q 29$ genes, we found several genes that have been extensively implicated in neuropsychiatric disease (Table S2.1). These include MECP2, NRXN1, GRIN2A, GRIN2B, CHD8, SATB2, CNTNAP2, FOXP1, PTEN, and SCN2A. Motivated by this observation, we asked whether top neighbors of individual 3q29 genes significantly overlap with known SZ, ASD, or IDD-risk genes (Fig. 3a). We curated six evidence-based lists of SZ [100-104], ASD [105,106,], and IDD-risk genes [107], which span loci across a wide range of the allele frequency spectrum and include postmortem findings from case-control gene expression studies (Table S2.2). 3q29 genes whose top neighbors showed an overrepresentation of SZ, ASD, and/or IDD risk genes (adjusted $P<0.05$ ) were subsequently prioritized as likely genetic drivers of neuropsychiatric risk in 3q29Del syndrome, along with their SZ, ASD, and/or IDD-related top neighbors from the enrichment findings (Fig. 3a). We found overrepresentation of one or more established risk gene sets among the top neighbors of nine 3q29 genes (Benjamini-Hochberg adjusted $P<0.05$ ) (Fig. 3b and Table S2.3).

To evaluate the specificity of the identified patterns of polygenic disease burden, we also tested these top neighbors for overlap with known Parkinson's disease (PD) [108], late-onset Alzheimer's disease (AD) [109], and inflammatory bowel disease (IBD) risk genes [110] (Table S2.2). These phenotypes have no known link to 3q29Del, thus, their risk loci were considered negative controls. Common variants associated with height [111] (Table S2.2) were included as a fourth negative control to rule out a potential bias associated with large differences in the sizes of curated gene sets.

Concurrently, we found no statistically significant evidence for overrepresentation of $A D$ or IBD-risk genes among the interrogated top neighbors (Fig. 3b). Only the top neighbors of SENP5 showed a significant overlap with height-associated genes (adjusted $P=2.36 \mathrm{E}-02$ ), and the top neighbors of NRROS, which did not show an enrichment for known IDD, ASD, or SZ risk genes, exhibited a small but significant overlap with known PD-risk genes (adjusted $P=2.00 \mathrm{E}-02$ ) (Fig. 3b). Overall, 19 out of 96 hypergeometric tests $(20 \%)$ revealed a significant overrepresentation of SZ, ASD, and/or IDD-risk gene sets among the top neighbors of 3q29 genes. By contrast, only 2 out of 64 (3\%) hypergeometric tests indicated a significant overlap with the negative control gene sets. The substantial margin between these two enrichment ratios supports the high specificity of our network-derived inferences for uncovering biology relevant to 3q29Del. By leveraging guilt by association, we prioritize BDH1, CEP19, DLG1, FBXO45, PIGZ, RNF168, SENP5, UBXN7, and WDR53, along with their 284 unique $\mathrm{SZ}, \mathrm{ASD}$, and/or IDD-related top neighbors from significant overlap tests as likely drivers of the neuropsychiatric consequences of 3q29Del (Fig. 4a and Table S2.3).

\section{Disease-relevant driver genes prioritized by network analysis load onto key biological pathways linked to neuropsychiatric disorders}

To formulate testable hypotheses about the biological mechanisms linking the 3q29 locus to neuropsychiatric phenotypes, we interrogated whether the prioritized driver genes identified in our network analysis assemble into known biological pathways. Functional enrichment analysis on the union of 293 prioritized driver genes (including nine 3q29 genes) revealed significant overrepresentation of eight biological pathways annotated by the Reactome and KEGG databases (Fig. $4 \mathrm{~b}$ and Table S2.4). These include axon guidance (adjusted $P=3.64 \mathrm{E}-03$ ), long-term potentiation (adjusted $P=7.29 \mathrm{E}-03$ ), and regulation of actin cytoskeleton (adjusted $P=1.17 \mathrm{E}-02$ ). Additionally, several GO biological processes (GO:BP), including chromosome organization (adjusted $P=3.81 \mathrm{E}-$ 09 ), histone modification (adjusted $P=3.31 \mathrm{E}-08$ ), neuron differentiation (adjusted $P=1.88 \mathrm{E}-04$ ), neurogenesis (adjusted $P=1.89 \mathrm{E}-$ 03 ), and excitatory postsynaptic potential (adjusted $P=8.97 \mathrm{E}-03$ ) were overrepresented among the predicted drivers (Fig. 4b, $c$ and Table S2.4). We hypothesize that the disruption of one or more of these biological pathways and processes, some of which have been demonstrated to be altered in idiopathic SZ and ASD [2,112,], lie on the causal pathway to neuropsychopathology in 3q29Del syndrome. For extended results, see Supplemental Results.

\section{Network-derived targets predict differentially expressed genes in the mouse model of 3q29Del}

Perturbation of $3 q 29$ gene dosage in neural tissue is expected to lead to the differential expression of the true transcriptomic network partners of $3 q 29$ genes. Following this premise, we tested the enrichment of the network targets identified in this study for differential expression in Del16 ${ }^{+/ B d h 1-T f r c}$ mice compared with WT littermates. RNA-Seq analysis revealed 290 protein-coding DEGs with known human homologs $(P<0.05), 17$ of which were identified as 3q29 interval genes (Bdh1, Cep19, Dlg1, Fbxo45, Mfi2, Ncbp2, Nrros, Pak2, Pcyt1a, Pigx, Pigz, Rnf168, Senp5, Tctex1d2, Tfrc, Ubxn7, and Wdr53) (Fig. 5a, b and Table S2.5). The scaled expression of these $3 q 29$ genes showed a consistent reduction proportional to gene copy number (Fig. 5a and Table S2.5). 

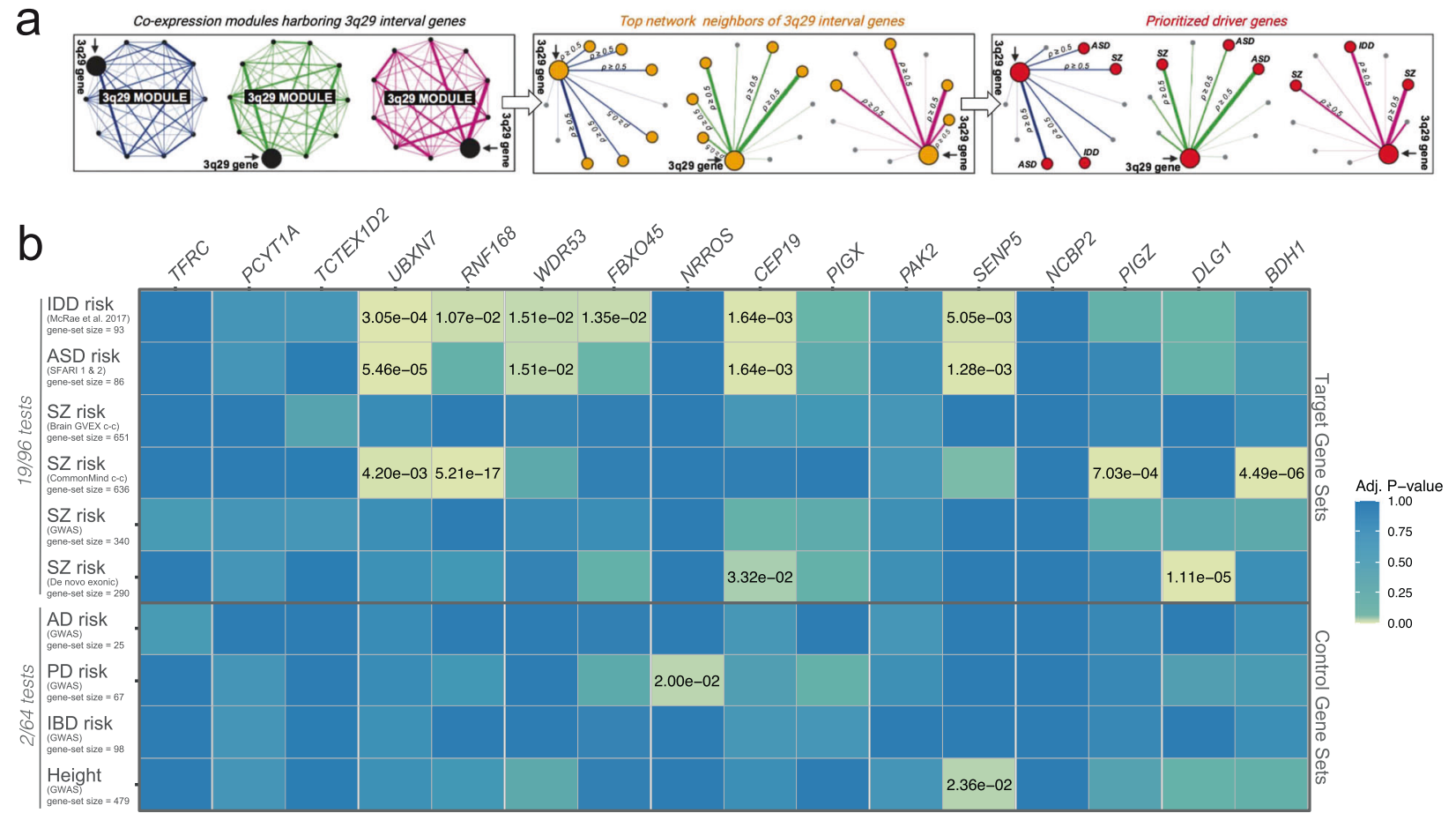

Fig. 3 3q29 interval genes form transcriptomic subnetworks enriched for known schizophrenia, autism, and intellectual/developmental disability-risk genes. a Schematic of strategy to test the neuropsychiatric disease burden associated with top network neighbors of $3 q 29$ interval genes and to refine a list of prioritized driver genes. To minimize false positives, 3q29 modules were reduced to strongly connected top neighbors (yellow nodes) of individual 3q29 genes, which were then screened for a significant overlap with known risk genes (red nodes) for schizophrenia (SZ), autism spectrum disorders (ASD), and intellectual/developmental disability (IDD), spanning known associations over a wide spectrum of allele frequencies. A top neighbor was defined as any node whose gene expression profile had a moderate-to-high pairwise correlation (Spearman's rho $(\rho) \geq 0.5, P<0.05$ ) with a 3q29 interval gene within the same module. By leveraging the guilt by association principle, the 3q29 interval genes that showed a significant enrichment of known SZ, ASD, and/or IDD risk genes among their respective top neighbors were prioritized as likely drivers of the neurodevelopmental and psychiatric consequences of 3q29Del, along with their SZ, ASD, and/or IDD-related top neighbors from significant overlap tests. b Adjusted $p$ values from hypergeometric tests identifying the significance of the overlap between top neighbors of individual 3q29 genes and known risk genes for SZ, ASD, and IDD. Risk gene sets for three traits with no known association to the 3q29Del syndrome were also tested for overrepresentation as negative controls. Common variants associated with height were included as another negative control to rule out a potential bias introduced by gene-set size. Nine protein-coding genes from the 3 q29 interval formed transcriptomic subnetworks that are significantly enriched for known SZ, ASD, and/or IDD risk genes (orange highlight, adjusted $P<0.05)$. The proportion of hypergeometric tests with significant overrepresentation of SZ, ASD, and IDD gene sets (19/96) was found to be an order of magnitude larger than that of the negative control tests (2/64), demonstrating the high specificity of the identified enrichment patterns for reported 3q29Del-associated phenotypes.

All 290 DEGs were tested for enrichment of network-derived targets identified via WGCNA at three scales of network interconnectedness: (i) broad 3q29 network (11,924 genes), (ii) topneighbor-based 3q29 subnetwork (5087 genes), and (iii) prioritized drivers (280 genes). Hypergeometric tests revealed significant enrichment of the interrogated DEGs for network-derived ties at all three levels of this analysis $(P<0.05$; Fig. 5b). The list of DEGs, including the subsets intersecting WGCNA-derived targets, and the list of genes corresponding to the three levels of network interconnectedness interrogated in this analysis are provided in Table S2.5. See Supplemental Results for extended results.

\section{DISCUSSION}

The 3q29Del has been reliably associated with extraordinary risk for serious neuropsychiatric illness and therefore may offer key insights to advance our understanding of the biological basis of these complex disorders. Currently, the driver genes and affected biological pathways that link 3q29Del to neuropsychiatric pathology remain unknown. To avoid bias introduced by annotation-based criteria in the formulation of mechanistic hypotheses, we engaged a system-level vantage point and interrogated the collective behavior of 3q29 interval genes with the global protein-coding transcriptome of the healthy human brain. We leveraged publicly available transcriptomic data from the GTEx Project [58] to perform WGCNA [29,42,] on postmortem cortical samples from donors with no known history of psychiatric or neurological disease. We focused our analysis on the adult PFC and analyzed the resulting network to identify the modular properties and undirected connectivity patterns of the 3q29 interval, which yielded key predictions into interrelated functions and disease associations. Finally, we assessed the validity of our graph-based predictions in an experimental system by conducting RNA-sequencing in mice harboring a homologous deletion to the human 3q29Del locus [20]. Our findings provide foundational information to formulate rigorous, targeted, and testable hypotheses on the causal drivers and mechanisms underlying the largest known single genetic risk factor for SZ.

Genomic studies have identified several recurrent CNVs that confer high risk for neuropsychiatric disorders $[2,10$,$] . The current$ challenge is to understand which genes within these loci are the major drivers of risk. In the 3q29 locus, DLG1 and PAK2 have been most often proposed as candidate drivers of neuropsychiatric phenotypes [12,25,113,]. Indeed, a recent literature search revealed more publications related to these genes than of all other 3q29Del genes combined (Fig. S6). Consistent with previous reports of an association between DLG1 and SZ [18,19,], the current study presents network-level evidence for prioritizing 
a
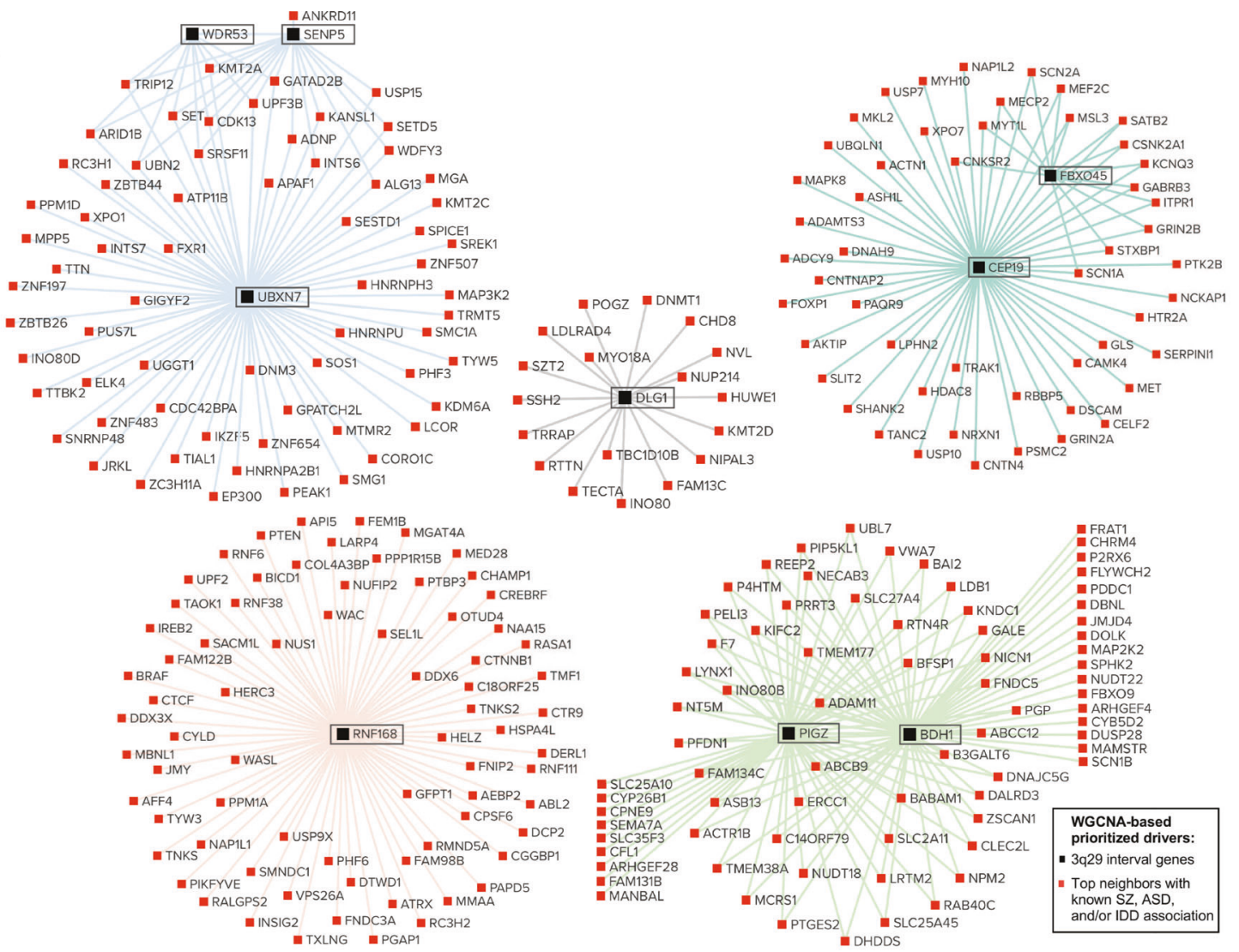

b

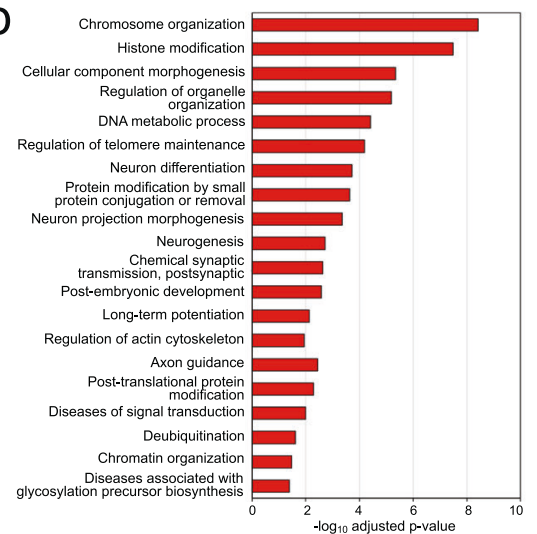

C

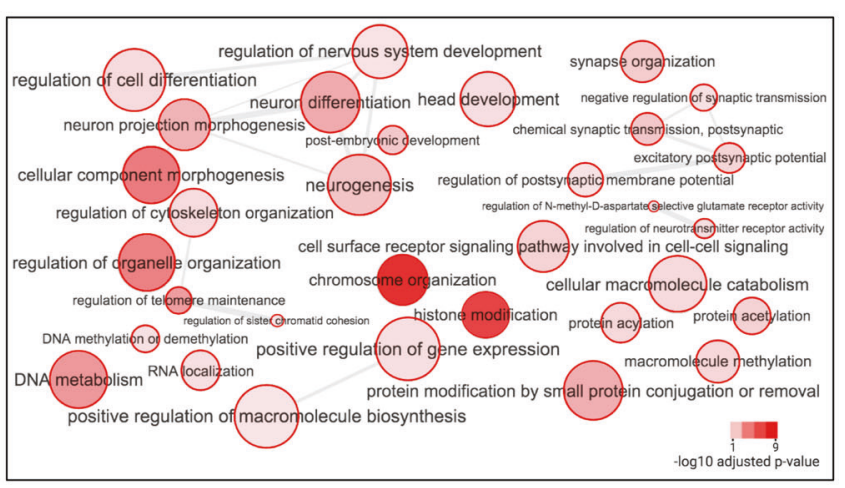

Fig. 4 Network of prioritized drivers predicted to contribute to the neuropsychiatric sequelae of 3q29Del. a Nine protein-coding genes from the 3q29 interval formed top-neighbor-based transcriptomic subnetworks that were significantly enriched for known schizophrenia (SZ), autism spectrum disorder (ASD), and intellectual/developmental disability (IDD)-risk genes (adjusted $P<0.05$ ). Black and red nodes illustrated in this network diagram represent these nine $3 q 29$ genes and their 284 top neighbors with known SZ, ASD, and/or IDD-association, respectively. The union of these prioritized genes constitute 293 genetic drivers predicted to contribute to the neurodevelopmental and psychiatric phenotypes of 3q29Del. The color of network edges that connect node-pairs represents module assignment. $\mathbf{b}$ Top 20 biological processes and pathways with significant enrichment among prioritized drivers (adjusted $P<0.05$ ). The identified Gene Ontology biological processes (GO: BP) and Reactome and KEGG biological pathways point to key mechanisms through which select genes within the 3q29Del locus and their likely partners outside the interval are predicted to influence susceptibility to SZ, ASD, and IDD. c Organization of all statistically significant biological processes enriched in prioritized drivers into a network of related functional annotation categories. GO:BP terms are connected if they have a high overlap (share many genes); edge width represents magnitude of the overlap.

DLG1 as a neuropsychiatric disease-linked gene. Surprisingly, however, our analysis does not support inclusion of PAK2 as a predicted driver of neuropsychiatric risk. Instead, our results lend support to DLG1 and eight other 3q29 genes, most of which are largely understudied, as key players in 3q29Del syndrome. Our unbiased approach prioritizes BDH1, CEP19, DLG1, FBXO45, PIGZ, RNF168, SENP5, UBXN7, and WDR53 as primary drivers.
It is currently unknown whether the biological basis of neuropsychiatric risk associated with recurrent CNVs overlaps with that of individuals who share the same clinical diagnosis but do not share the same rare genetic variant. Our findings suggest that molecular perturbations caused by the hemizygous deletion of select $3 q 29$ genes may overlap with the genetic etiologies contributing to idiopathic forms of SZ, ASD, and IDD. Disease-relevant driver genes 

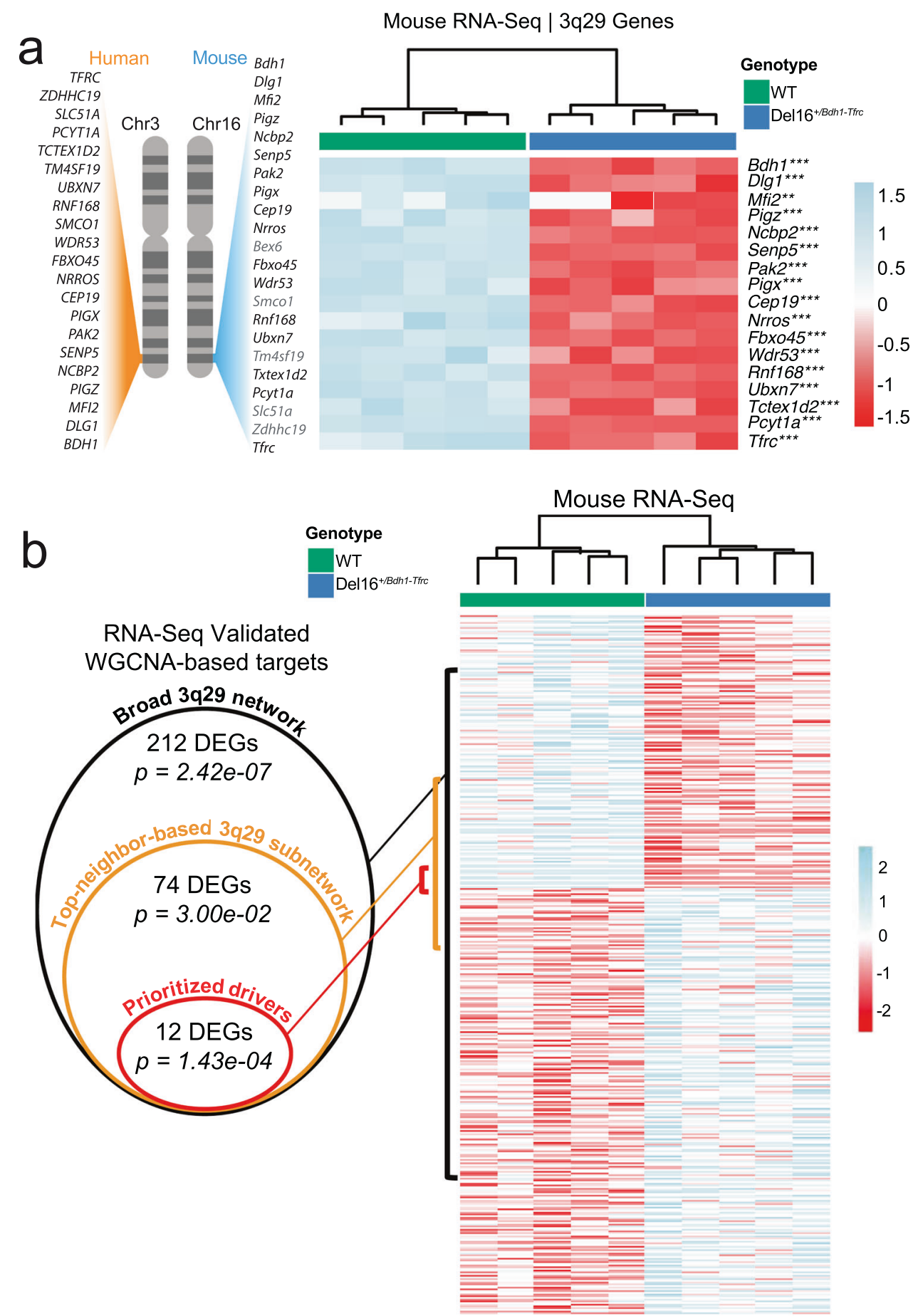

Fig. 5 WGCNA network predicts differentially expressed genes in the mouse model of 3q29Del. a Gene-scaled expression of all detected protein-coding genes within the homologous 3 q29 interval shows a consistent reduction in Del16 ${ }^{+/ B d h 1-T f r c}$ mice relative to wild-type (WT) littermates, proportional to gene copy number. Genes in gray were not detected by RNA-Seq. Asterisks indicate level of significance (***P< 0.0001 , ** $P<0.001)$. b 290 protein-coding genes with known mouse-human homologs were found to be differentially expressed $(P<0.05)$ in Del16 $16^{+/ B d h} 1$-Tfrc mice relative to wild-type (WT) littermates. These DEGs were tested for enrichment of genes found in the broad $3 q 29$ network (all genes in 3q29 modules), top-neighbor based 3q29 subnetwork, and disease-associated prioritized drivers. A significant enrichment was found at each level of network interconnectedness $(P<0.05)$. Upregulated genes are in blue and downregulated genes are in red.

prioritized by our network analysis are enriched for canonical biological pathways, such as neurogenesis, neuron differentiation, synapse organization, excitatory postsynaptic potential, long-term potentiation, axon guidance, regulation of actin cytoskeleton, signal transduction, posttranslational protein modifications, chromatin organization, and histone modification. We hypothesize that the disruption of one or more of these biological processes, some of which are altered in idiopathic SZ and ASD [2,112,], lie on the causal pathway to neuropsychopathology in 3q29Del syndrome.

No single gene within the interval has been definitively associated with neuropsychiatric disease, prompting the hypothesis that neuropathology in 3q29Del emerges upon loss of multiple genes that are functionally connected. While a single nucleotide polymorphism in DLG1 has been associated with SZ in 
a case-control study $[18,19$,$] , the risk associated with this variant$ does not approach that of 3q29Del, suggesting that the neuropsychiatric risk associated with this CNV is distributed across more than one gene in the locus. To investigate functional connections across multiple 3q29 genes, we conducted an unsupervised analysis of the modular organization of the adult human PFC. We found the $213 q 29$ genes distributed into three meta-modules and seven modules, with 18 genes converging into just four modules. Hence, 3q29 genes display both distributed and convergent effects in the adult human cortical transcriptome. Rather than functioning as independent agents, sets of 3 q29 genes may have shared and/or synchronized function and constitute interacting sources of pathology. It is conceivable that the consequences of the haploinsufficiency arise through the weakening of multiple distinct pathways that normally provide protective redundancies (distributed model), and/or through multiple insults to a functionally connected module that cumulatively disrupt resiliency (convergent biology model). These hypotheses warrant further testing.

A major goal of this study was to infer unknown functions for understudied 3q29 genes by leveraging well-studied co-clustering genes. Pathway analysis of modules harboring $3 q 29$ genes revealed likely functional involvement of the 3q29 locus in not only nervous-system specific functions, but also in core aspects of cell biology that are nonspecific to an organ system. The closelyrelated black and midnight-blue modules were significantly associated with regulation of gene expression, chromatin organization, and DNA repair. The green and turquoise modules were both associated with nervous system development and function, and in particular, regulation and organization of synaptic signaling and components. This finding is surprising because most of the 3 q29 genes located in these latter two modules have not been identified as synaptic genes. Similarly, the 3q29 genes in the black and midnight-blue modules have not been implicated in gene regulatory pathways or DNA repair. We maintain that biological functions of poorly annotated genes can be inferred through the graph-based modeling of inter-gene relationships. Thereby, we predict novel roles for individual 3q29 genes in functions related to synaptic transmission, modulation of neurotransmission, synapse structure and function, mitochondrial metabolism, transcriptional and translational regulation, chromatin remodeling, cell cycle regulation, and protein modification, localization and turnover. We propose that the subset of predicted functions that are nonspecific to an organ system likely contribute to global developmental outcomes in 3q29Del.

Analysis of eigengene-based connectivity revealed that UBXN7 is a hub gene, with top neighbors enriched for known association with all three major neuropsychiatric phenotypes of 3q29Del. Hub nodes of biological networks are often associated with human disease [114,115,]. Disease-genes, identified from OMIM's Morbid Map of the Human Genome, disproportionately exhibit hub-gene characteristics, with protein products participating in more known PPIs than that of non-disease genes [116]. Supported by this literature, we predict that (1) UBXN7 exerts critical influence on a large network of co-expressed genes, and (2) loss-of-function (LoF) mutations in UBXN7 can cause major dysfunction in affiliated biological pathways (indeed, its pLI score $=0.99$, i.e., extremely intolerant to LoF [117]). We prioritize UBXN7 as a major driver in 3q29Del syndrome. UBXN7 has not been previously linked to neuropsychiatric disorders or proposed as a candidate driver of 3q29Del syndrome. However, UBXN7 has been reported to regulate the ASD-associated E3 ubiquitin ligase Cullin-3 (CUL3) [118], an interaction that deserves more attention in light of our findings [97]. In fact, UBXN7 is one of three genes involved in the ubiquitin-proteasome system (UPS)-along with RNF168 and FBXO45-that were prioritized in our analysis. Accumulating evidence indicates multiple links between the UPS and SZ, though the causal relationship is still unclear (reviewed by [119]).
Our analysis indicates that the UPS may be disrupted at multiple levels by haploinsufficiency of these three genes in 3q29Del.

The network described here was built from adult human PFC gene expression data, while the experimental system used to test the validity of the identified network targets was the mouse model of 3q29Del at postnatal day 7 [20], which most closely matches the perinatal stage of brain development in humans [120]. Notwithstanding this considerable difference in developmental phase, we found significant enrichment of networkderived targets among DEGs identified in this model, presenting proof of concept for the validity of our network analysis approach for uncovering biologically meaningful associations. These results also indicate that a significant fraction of transcriptomic network connections formed by the 3q29 locus may be relatively stable through development and are evolutionarily conserved in mice.

One limitation of the current study is its singular focus on protein-coding elements. How the noncoding elements of the interval, along with splice variants, integrate into the predictions formulated in this study is ripe for future investigation. Overall, the transcriptomic network identified in this study is predicted to connect 3q29 interval genes with gene sets outside the interval that participate in the same or overlapping biological process and associate with similar disease phenotypes. Perturbation of $3 q 29$ interval gene dosage is expected to also perturb the functioning of network-partners outside the recurrent 3q29Del locus. However, note that the underlying structure of weighted gene coexpression networks is agnostic to the mechanistic order of cellular and molecular events. The information necessary to derive the order of biological interactions is not an explicit outcome of gene co-expression itself, since such inferences require timedependent analysis of combinatorial interactions between nodes. As a result, some of the network partners identified in this study are expected to function upstream of their co-expressed 3q29 gene partner and would likely not be affected by 3q29Del. Simultaneously, this direction-agnostic property also suggests that network-based predictions formulated in this study are likely relevant to biological pathways and processes implicated in 3q29 duplication syndrome [121], which was recently shown to manifest phenotypic concordance with 3q29Del syndrome in multiple clinical areas, similar to relationships identified in other reciprocal CNV disorders [122].

Moreover, the complex interactions between molecules can be dynamic across time and space [56]. Hence, a future direction will be to ask whether the network connections formed by $3 q 29$ interval genes in the adult PFC show differential expression in the neural tissue of 3q29Del carriers and whether they show temporal and/or spatial variation.

Finally, our analysis does not preclude the possibility that other $3 q 29$ interval genes moderate phenotypic expressivity. For example, while the dark turquoise module (including PAK2) did not harbor prioritized driver genes, it was significantly associated with estrogen receptor-mediated signaling. An intriguing emerging feature of 3q29Del syndrome is the markedly reduced sex bias in risk for ASD [9]. Additional studies will be required to assess the drivers of sex-specific phenotypes of 3q29Del syndrome.

Now that recurrent, highly penetrant CNV loci have been identified as important risk factors for neuropsychiatric disorders, determination of the component genes driving this risk is the next step toward deciphering mechanisms. We used an unbiased systems biology approach that leveraged the power of open data to infer unknown functions for understudied 3q29 interval genes, and to refine the $3 q 29$ locus to nine prioritized driver genes, including one hub gene. Importantly, this approach can partially overcome barriers to formulation of relevant hypotheses that are introduced by poor annotation of interval genes, without requiring laborious, expensive, and time-consuming experiments to functionally characterize all genes within the interval. Our results reveal the power of this approach for prioritization of 
putative drivers. Ongoing and future studies will be directed at understanding how these genes work in concert and how multiple haploinsufficiencies confer risk for neuropsychiatric disease.

\section{REFERENCES}

1. Levinson DF, Duan J, Oh S, Wang K, Sanders AR, Shi J, et al. Copy number variants in schizophrenia: confirmation of five previous findings and new evidence for 3q29 microdeletions and VIPR2 duplications. Am J Psychiatry. 2011;168:302-16.

2. Marshall CR, Howrigan DP, Merico D, Thiruvahindrapuram B, Wu W, Greer DS, et al. Contribution of copy number variants to schizophrenia from a genomewide study of 41,321 subjects. Nat Genet. 2017;49:27-35.

3. Mulle JG, Dodd AF, McGrath JA, Wolyniec PS, Mitchell AA, Shetty AC, et al. Microdeletions of $3 q 29$ confer high risk for schizophrenia. Am J Hum Genet. 2010;87:229-36.

4. Szatkiewicz JP, O'Dushlaine C, Chen G, Chambert K, Moran JL, Neale BM, et al. Copy number variation in schizophrenia in Sweden. Mol Psychiatry. 2014;19:762-73.

5. Mulle JG. The 3q29 deletion confers $>40$-fold increase in risk for schizophrenia. Mol Psychiatry. 2015;20:1028-9.

6. Russo RS, Gambello MJ, Murphy MM, Aberizk K, Black E, Burrell TL, et al. Deep phenotyping in $3 q 29$ deletion syndrome: recommendations for clinical care. Genet Med. 2021. https://doi.org/10.1038/s41436-020-01053-1.

7. Glassford MR, Rosenfeld JA, Freedman AA, Zwick ME, Mulle JG, Unique Rare Chromosome Disorder Support G. Novel features of 3q29 deletion syndrome: results from the 3q29 registry. Am J Med Genet A. 2016;170A:999-1006.

8. Cox DM, Butler MG. A clinical case report and literature review of the $3 q 29$ microdeletion syndrome. Clin Dysmorphol. 2015;24:89-94.

9. Pollak RM, Murphy MM, Epstein MP, Zwick ME, Klaiman C, Saulnier CA, et al. Neuropsychiatric phenotypes and a distinct constellation of ASD features in $3 q 29$ deletion syndrome: results from the 3q29 registry. Mol Autism. 2019;10:30.

10. Sanders SJ, He X, Willsey AJ, Ercan-Sencicek AG, Samocha KE, Cicek AE, et al. Insights into autism spectrum disorder genomic architecture and biology from 71 risk loci. Neuron. 2015;87:1215-33.

11. Singh MD, Jensen $M$, Lasser M, Huber E, Yusuff T, Pizzo L, et al. NCBP2 modulates neurodevelopmental defects of the $3 q 29$ deletion in Drosophila and Xenopus laevis models. PLoS Genet. 2020;16:e1008590.

12. Carroll LS, Williams HJ, Walters J, Kirov G, O'Donovan MC, Owen MJ. Mutation screening of the 3q29 microdeletion syndrome candidate genes DLG1 and PAK2 in schizophrenia. Am J Med Genet B Neuropsychiatr Genet. 2011;156B:844-9.

13. Gardoni F, Mauceri D, Fiorentini C, Bellone C, Missale C, Cattabeni F, et al. CaMKII-dependent phosphorylation regulates SAP97/NR2A interaction. J Biol Chem. 2003;278:44745-52.

14. Leonard AS, Davare MA, Horne MC, Garner CC, Hell JW. SAP97 is associated with the alpha-amino-3-hydroxy-5-methylisoxazole-4-propionic acid receptor GluR1 subunit. J Biol Chem. 1998;273:19518-24.

15. Lin El, Jeyifous O, Green WN. CASK regulates SAP97 conformation and its interactions with AMPA and NMDA receptors. J Neurosci. 2013;33:12067-76.

16. Zhou W, Zhang L, Guoxiang X, Mojsilovic-Petrovic J, Takamaya K, Sattler R, et al. GluR1 controls dendrite growth through its binding partner, SAP97. J Neurosci. 2008;28:10220-33.

17. Moghaddam B, Javitt D. From revolution to evolution: the glutamate hypothesis of schizophrenia and its implication for treatment. Neuropsychopharmacol. 2012;37:4-15.

18. Uezato A, Kimura-Sato J, Yamamoto N, lijima Y, Kunugi H, Nishikawa T. Further evidence for a male-selective genetic association of synapse-associated protein 97 (SAP97) gene with schizophrenia. Behav Brain Funct. 2012;8:2.

19. Uezato A, Yamamoto N, Jitoku D, Haramo E, Hiraaki E, Iwayama Y, et al. Genetic and molecular risk factors within the newly identified primate-specific exon of the SAP97/DLG1 gene in the 3q29 schizophrenia-associated locus. Am J Med Genet B Neuropsychiatr. Genet. 2017;174:798-807.

20. Rutkowski TP, et al. Behavioral changes and growth deficits in a CRISPR engineered mouse model of the schizophrenia-associated 3q29 deletion. Mol Psychiatry. 2019. https://doi.org/10.1038/s41380-019-0413-5.

21. Bokoch GM. Biology of the p21-activated kinases. Annu Rev Biochem. 2003;72:743-81.

22. Wang Y, Zeng C, Li J, Zhou Z, Ju X, Xia S, et al. PAK2 haploinsufficiency results in synaptic cytoskeleton impairment and autism-related behavior. Cell Rep. 2018;24:2029-41.

23. Allen KM, Gleeson JG, Bagrodia S, Partington MW, MacMillan JC, Cerione RA, et al. PAK3 mutation in nonsyndromic X-linked mental retardation. Nat. Genet. 1998;20:25-30.
24. Tarpey P, Parnau J, Blow M, Woffendin H, Bignell G, Cox C, et al. Mutations in the DLG3 gene cause nonsyndromic X-linked mental retardation. Am J Hum Genet. 2004;75:318-24.

25. Grice SJ, Liu JL, Webber C. Synergistic interactions between Drosophila orthologues of genes spanned by de novo human CNVs support multiple-hit models of autism. PLoS Genet. 2015;11:e1004998.

26. Pabis M, Neufeld N, Shav-Tal Y, Neugebauer KM. Binding properties and dynamic localization of an alternative isoform of the cap-binding complex subunit CBP20. Nucleus. 2010;1:412-21.

27. Johnson EC, Border R, Melroy-Greif WE, de Leeuw CA, Ehringer MA, Keller MC. No evidence that schizophrenia candidate genes are more associated with schizophrenia than noncandidate genes. Biol Psychiatry. 2017;82:702-8.

28. Eisenberg D, Marcotte EM, Xenarios I, Yeates TO. Protein function in the postgenomic era. Nature. 2000;405:823-6.

29. Zhang B, Horvath S. A general framework for weighted gene co-expression network analysis. Stat Appl Genet Mol Biol. 2005;4:Article17.

30. Ma'ayan A, Blitzer RD, lyengar R. Toward predictive models of mammalian cells. Annu Rev Biophys Biomol Struct. 2005;34:319-49.

31. Barabasi AL, Gulbahce N, Loscalzo J. Network medicine: a network-based approach to human disease. Nat Rev Genet. 2011;12:56-68.

32. Qiu Y, Arbogast T, Lorenzo SM, Li H, Tang SC, Richardson E, et al. Oligogenic effects of 16 p11.2 copy-number variation on craniofacial development. Cell Rep. 2019;28:3320-8 e3324.

33. Singer GA, Lloyd AT, Huminiecki LB, Wolfe KH. Clusters of co-expressed genes in mammalian genomes are conserved by natural selection. Mol Biol Evol. 2005;22:767-75.

34. de la Fuente A. From 'differential expression' to 'differential networking' identification of dysfunctional regulatory networks in diseases. Trends Genet. 2010;26:326-33.

35. Wolfe CJ, Kohane IS, Butte AJ. Systematic survey reveals general applicability of "guilt-by-association" within gene coexpression networks. BMC Bioinforma. 2005;6:227.

36. Horan K, Jang C, Bailey-Serres J, Mittler R, Shelton C, Harper JF, et al. Annotating genes of known and unknown function by large-scale coexpression analysis. Plant Physiol. 2008;147:41-57.

37. Ahn AC, Tewari M, Poon CS, Phillips RS. The limits of reductionism in medicine: could systems biology offer an alternative? PLoS Med. 2006;3:e208.

38. Oliver S. Guilt-by-association goes global. Nature. 2000;403:601-3.

39. Lee HK, Hsu AK, Sajdak J, Qin J, Pavlidis P. Coexpression analysis of human genes across many microarray data sets. Genome Res. 2004;14:1085-94.

40. Moreau Y, Tranchevent LC. Computational tools for prioritizing candidate genes: boosting disease gene discovery. Nat Rev Genet. 2012;13:523-36.

41. Oti M, Brunner HG. The modular nature of genetic diseases. Clin Genet. 2007;71:1-11.

42. Langfelder P, Horvath S. WGCNA: an R package for weighted correlation network analysis. BMC Bioinforma. 2008;9:559.

43. Radulescu E, Jaffe AE, Straub RE, Chen Q, Shin JH, Hyde TM, et al. Identification and prioritization of gene sets associated with schizophrenia risk by coexpression network analysis in human brain. Mol Psychiatry. 2020;25:791-804.

44. Etemadikhah M, Niazi A, Wetterberg L, Feuk L. Transcriptome analysis of fibroblasts from schizophrenia patients reveals differential expression of schizophrenia-related genes. Sci. Rep. 2020;10:630.

45. Gordon A, Forsingdal A, Klewe IV, Nielsen J, Didriksen M, Werge T, et al. Transcriptomic networks implicate neuronal energetic abnormalities in three mouse models harboring autism and schizophrenia-associated mutations. Mol Psychiatry. 2019. https://doi.org/10.1038/s41380-019-0576-0.

46. Chen C, Cheng L, Grennan K, Pibiri F, Zhang C, Badner JA, et al. Two gene coexpression modules differentiate psychotics and controls. Mol Psychiatry. 2013;18:1308-14.

47. Oron O, Getselter D, Shohat S, Reuveni E, Lukic I, Shifman S, et al. Gene network analysis reveals a role for striatal glutamatergic receptors in dysregulated risk-assessment behavior of autism mouse models. Transl Psychiatry. 2019;9:257.

48. Lin M, Pedrosa E, Hrabovsky A, Chen J, Puliafito BR, Gilbert SR, et al. Integrative transcriptome network analysis of iPSC-derived neurons from schizophrenia and schizoaffective disorder patients with 22q11.2 deletion. BMC Syst. Biol. 2016;10:105.

49. Brennand K, Savas JN, Kim Y, Tran N, Simone A, Hashimoto-Torii K, et al. Phenotypic differences in hiPSC NPCs derived from patients with schizophrenia. Mol Psychiatry. 2015;20:361-8.

50. Steinberg J, Webber C. The roles of FMRP-regulated genes in autism spectrum disorder: single- and multiple-hit genetic etiologies. Am J Hum Genet. 2013;93:825-39.

51. Jalbrzikowski M, Lazaro MT, Gao F, Huang A, Chow C, Geschwind DH, et al. Transcriptome profiling of peripheral blood in 22q11.2 deletion syndrome 
reveals functional pathways related to psychosis and autism spectrum disorder. PLOS ONE. 2015;10:e0132542.

52. Wang P, Zhao D, Lachman HM, Zheng D. Enriched expression of genes associated with autism spectrum disorders in human inhibitory neurons. Trans/ Psychiatry. 2018;8:13.

53. Maschietto M, Tahira AC, Puga R, Lima L, Mariani D, Paulsen Bda S, et al. Coexpression network of neural-differentiation genes shows specific pattern in schizophrenia. BMC Med Genomics. 2015;8:23.

54. Hafner H, Maurer K, Löffler W, Fätkenheuer B, Heiden W an der, Riecher-Rössler $A$, et al. The epidemiology of early schizophrenia. Influence of age and gender on onset and early course. Br J Psychiatry. 1994;23:29-38.

55. Howard R, Rabins PV, Seeman MV, Jeste DV. Late-onset schizophrenia and verylate-onset schizophrenia-like psychosis: an international consensus. The International Late-Onset Schizophrenia Group. Am J Psychiatry. 2000;157:172-8.

56. Kang HJ, Kawasawa Yl, Cheng F, Zhu Y, Xu X, Li M, et al. Spatio-temporal transcriptome of the human brain. Nature. 2011;478:483-9.

57. Fuster JM. Frontal lobe and cognitive development. J Neurocytol. 2002;31:373-85.

58. Consortium GT. Human genomics. The genotype-tissue expression (GTEx) pilot analysis: multitissue gene regulation in humans. Science. 2015;348:648-60.

59. Leek JT, Johnson WE, Parker HS, Jaffe AE, Storey JD. The sva package for removing batch effects and other unwanted variation in high-throughput experiments. Bioinformatics. 2012;28:882-3.

60. Johnson WE, Li C, Rabinovic A. Adjusting batch effects in microarray expression data using empirical Bayes methods. Biostatistics. 2007;8:118-27.

61. Wilcox R. Introduction to robust estimation and hypothesis testing. Academic Press; 1997.

62. Song $L$, Langfelder $P$, Horvath $S$. Comparison of co-expression measures: mutual information, correlation, and model based indices. BMC Bioinforma. 2012;13:328.

63. Barabasi AL, Oltvai ZN. Network biology: understanding the cell's functional organization. Nat Rev Genet. 2004;5:101-13.

64. Fu J, Keurentjes JJ, Bouwmeester H, America T, Verstappen FW, Ward JL, et al. System-wide molecular evidence for phenotypic buffering in Arabidopsis. Nat Genet. 2009;41:166-7.

65. Ouma WZ, Pogacar K, Grotewold E. Topological and statistical analyses of gene regulatory networks reveal unifying yet quantitatively different emergent properties. PLoS Comput Biol. 2018;14:e1006098.

66. Lachowiec J, Queitsch C, Kliebenstein DJ. Molecular mechanisms governing differential robustness of development and environmental responses in plants. Ann Bot. 2016;117:795-809.

67. Yip AM, Horvath S. Gene network interconnectedness and the generalized topological overlap measure. BMC Bioinforma. 2007;8:22

68. Ravasz E, Somera AL, Mongru DA, Oltvai ZN, Barabasi AL. Hierarchical organization of modularity in metabolic networks. Science. 2002;297:1551-5.

69. Langfelder $P$, Zhang B, Horvath S. Defining clusters from a hierarchical cluster tree: the Dynamic Tree Cut package for R. Bioinformatics. 2008;24:719-20.

70. Horvath S, Dong J. Geometric interpretation of gene coexpression network analysis. PLoS Comput Biol. 2008;4:e1000117.

71. Brainspan. Technical white paper: transcriptome profiling by RNA sequencing and exon microarray (v.5). 2013. Retrieved from https://help.brainmap.org/ download/attachments/3506181/Transcriptome_Profiling.pdf.

72. Langfelder $\mathrm{P}$, Luo R, Oldham MC, Horvath S. Is my network module preserved and reproducible? PLoS Comput Biol. 2011. https://doi.org/10.1371/journal. pcbi.1001057.

73. Reimand J, Isserlin R, Voisin V, Kucera M, Tannus-Lopes C, Rostamianfar A, et al. Pathway enrichment analysis and visualization of omics data using g:Profiler, GSEA, Cytoscape and EnrichmentMap. Nat Protoc. 2019;14:482-517.

74. Luck K, Kim DK, Lambourne L, Spirohn K, Begg BE, Bian W, et al. A reference map of the human binary protein interactome. Nature. 2020;580:402-8.

75. Szklarczyk D, Gable AL, Lyon D, Junge A, Wyder S, Huerta-Cepas J, et al. STRING v11: protein-protein association networks with increased coverage, supporting functional discovery in genome-wide experimental datasets. Nucleic Acids Res. 2019;47:D607-D613.

76. Ala U, Piro RM, Grassi E, Damasco C, Silengo L, Oti M, et al. Prediction of human disease genes by human-mouse conserved coexpression analysis. PLoS Comput Biol. 2008;4:e1000043.

77. Segal E, Friedman N, Koller D, Regev A. A module map showing conditional activity of expression modules in cancer. Nat Genet. 2004;36:1090-8.

78. Torkamani A, Dean B, Schork NJ, Thomas EA. Coexpression network analysis of neural tissue reveals perturbations in developmental processes in schizophrenia. Genome Res. 2010;20:403-12.

79. van Dam S, Cordeiro R, Craig T, van Dam J, Wood SH, de Magalhães JP. GeneFriends: an online co-expression analysis tool to identify novel gene targets for aging and complex diseases. BMC Genomics. 2012;13:535.
80. McCarroll SA, Murphy CT, Zou S, Pletcher SD, Chin CS, Jan YN, et al. Comparing genomic expression patterns across species identifies shared transcriptional profile in aging. Nat Genet. 2004;36:197-204.

81. Shen L, Sinai ISoMaM. GeneOverlap: Test and visualize gene overlaps v. R package version 1.20.0 2019. http://shenlab-sinai.github.io/shenlab-sinai.

82. Anders S, Pyl PT, Huber W. HTSeq-a Python framework to work with highthroughput sequencing data. Bioinformatics. 2015;31:166-9.

83. Love Ml, Huber W, Anders S. Moderated estimation of fold change and dispersion for RNA-seq data with DESeq2. Genome Biol. 2014;15:550.

84. Robinson MD, McCarthy DJ, Smyth GK. edgeR: a bioconductor package for differential expression analysis of digital gene expression data. Bioinformatics. 2010;26:139-40.

85. Sayers EW, Beck J, Bolton EE, Bourexis D, Brister JR, Canese K, et al. Database resources of the National Center for Biotechnology Information. Nucleic Acids Res. 2021;49:D10-D17.

86. Langfelder $\mathrm{P}$, Horvath $\mathrm{S}$. Eigengene networks for studying the relationships between co-expression modules. BMC Syst Biol. 2007;1:54

87. Mokhtari R, Lachman HM. The major histocompatibility complex (MHC) in schizophrenia: a review. J Clin Cell Immunol. 2016. https://doi.org/10.4172/21559899.1000479.

88. Sekar A, Bialas AR, de Rivera H, Davis A, Hammond TR, Kamitaki N, et al. Schizophrenia risk from complex variation of complement component 4. Nature. 2016;530:177-83.

89. Grigoriev A. On the number of protein-protein interactions in the yeast proteome. Nucleic Acids Res. 2003;31:4157-61.

90. Lander ES, Linton LM, Birren B, Nusbaum C, Zody MC, Baldwin J, et al. Initial sequencing and analysis of the human genome. Nature. 2001;409:860-921.

91. Hart GT, Ramani AK, Marcotte EM. How complete are current yeast and human protein-interaction networks? Genome Biol. 2006;7:120.

92. Huang $\mathrm{H}$, Jedynak BM, Bader JS. Where have all the interactions gone? Estimating the coverage of two-hybrid protein interaction maps. PLoS Comput Biol. 2007;3:e214.

93. Stumpf MP, Thorne T, de Silva E, Stewart R, An HJ, Lappe M, et al. Estimating the size of the human interactome. Proc Natl Acad Sci USA. 2008;105:6959-64.

94. Cooper TF, Morby AP, Gunn A, Schneider D. Effect of random and hub gene disruptions on environmental and mutational robustness in Escherichia coli. BMC Genomics. 2006;7:237.

95. Yu H, Braun P, Yildirim MA, Lemmens I, Venkatesan K, Sahalie J, et al. Highquality binary protein interaction map of the yeast interactome network. Science. 2008;322:104-10.

96. Alexandru G, Graumann J, Smith GT, Kolawa NJ, Fang R, Deshaies RJ. UBXD7 binds multiple ubiquitin ligases and implicates p97 in HIF1alpha turnover. Cell. 2008;134:804-16.

97. Tao S, Liu P, Luo G, Rojo de la Vega $M$, Chen $\mathrm{H}, \mathrm{Wu} T$, et al. p97 negatively regulates NRF2 by extracting ubiquitylated NRF2 from the KEAP1-CUL3 E3 complex. Mol Cell Biol. 2017. https://doi.org/10.1128/MCB.00660-16.

98. Uhlén M, Fagerberg L, Hallström BM, Lindskog C, Oksvold P, Mardinoglu A, et al. Proteomics. Tissue-based map of the human proteome. Science. 2015;347:1260419.

99. Chan SY, Loscalzo J. The emerging paradigm of network medicine in the study of human disease. Circ Res. 2012;111:359-74.

100. Fromer M, Roussos P, Sieberts SK, Johnson JS, Kavanagh DH, Perumal TM, et al. Gene expression elucidates functional impact of polygenic risk for schizophrenia. Nat Neurosci. 2016;19:1442-53.

101. Li J, Cai T, Jiang Y, Chen H, He X, Chen $C$, et al. Genes with de novo mutations are shared by four neuropsychiatric disorders discovered from NPdenovo database. Mol Psychiatry. 2016;21:290-7.

102. Meng $Q$, Wang K, Brunetti T, Xia Y, Jiao C, Dai R, et al. The DGCR5 long noncoding RNA may regulate expression of several schizophrenia-related genes. Sci Transl Med. 2018. https://doi.org/10.1126/scitranslmed.aat6912.

103. Akbarian S, Liu C, Knowles JA, Vaccarino FM, Farnham PJ, Crawford GE, et al. The PsychENCODE project. Nat Neurosci. 2015;18:1707-12.

104. Schizophrenia Working Group of the Psychiatric Genomics, C. Biological insights from 108 schizophrenia-associated genetic loci. Nature. 2014;511:421-7.

105. Banerjee-Basu S, Packer A. SFARI Gene: an evolving database for the autism research community. Dis Model Mech. 2010;3:133-5.

106. Abrahams BS, Arking DE, Campbell DB, Mefford HC, Morrow EM, Weiss LA, et al. SFARI gene 2.0: a community-driven knowledgebase for the autism spectrum disorders (ASDs). Mol Autism. 2013;4:36.

107. Deciphering Developmental Disorders, S. Prevalence and architecture of de novo mutations in developmental disorders. Nature. 2017;542:433-8.

108. Chang D, Nalls MA, Hallgrímsdóttir IB, Hunkapiller J, van der Brug M, Cai F, et al. A meta-analysis of genome-wide association studies identifies 17 new Parkinson's disease risk loci. Nat Genet. 2017;49:1511-6. 
109. Kunkle BW, Grenier-Boley B, Sims R, Bis JC, Damotte V, Naj AC, et al. Genetic meta-analysis of diagnosed Alzheimer's disease identifies new risk loci and implicates Abeta, tau, immunity and lipid processing. Nat Genet. 2019; 51:414-30.

110. Momozawa Y, Dmitrieva J, Théâtre E, Deffontaine V, Rahmouni S, Charloteaux B, et al. IBD risk loci are enriched in multigenic regulatory modules encompassing putative causative genes. Nat Commun. 2018;9:2427.

111. Yengo L, Sidorenko J, Kemper KE, Zheng Z, Wood AR, Weedon MN, et al. Metaanalysis of genome-wide association studies for height and body mass index in approximately 700000 individuals of European ancestry. Hum Mol Genet. 2018;27:3641-9.

112. Guan J, Cai JJ, Ji G, Sham PC. Commonality in dysregulated expression of gene sets in cortical brains of individuals with autism, schizophrenia, and bipolar disorder. Transl Psychiatry. 2019;9:152.

113. Willatt L, Cox J, Barber J, Cabanas ED, Collins A, Donnai D, et al. 3q29 microdeletion syndrome: clinical and molecular characterization of a new syndrome. Am J Hum Genet. 2005;77:154-60.

114. Wachi S, Yoneda K, Wu R. Interactome-transcriptome analysis reveals the high centrality of genes differentially expressed in lung cancer tissues. Bioinformatics. 2005;21:4205-8.

115. Jonsson PF, Bates PA. Global topological features of cancer proteins in the human interactome. Bioinformatics. 2006;22:2291-7.

116. Xu J, Li Y. Discovering disease-genes by topological features in human proteinprotein interaction network. Bioinformatics. 2006;22:2800-5.

117. Lek M, Karczewski KJ, Minikel EV, Samocha KE, Banks E, Fennell T, et al. Analysis of protein-coding genetic variation in 60,706 humans. Nature. 2016;536:285-91.

118. O'roak BJ, Vives L, Girirajan S, Karakoc E, Krumm N, Coe BP, et al. Sporadic autism exomes reveal a highly interconnected protein network of de novo mutations. Nature. 2012;485:246-50.

119. Luza S, Opazo CM, Bousman CA, Pantelis C, Bush Al, Everall IP. The ubiquitin proteasome system and schizophrenia. Lancet Psychiatry. 2020;7:528-37.

120. Semple BD, Blomgren K, Gimlin K, Ferriero DM, Noble-Haeusslein LJ. Brain development in rodents and humans: identifying benchmarks of maturation and vulnerability to injury across species. Prog Neurobiol. 2013;106-107:1-16.

121. Pollak RM, Zinsmeister MC, Murphy MM, Zwick ME, Emory 3q29 P, Mulle JG. New phenotypes associated with $3 q 29$ duplication syndrome: results from the 3q29 registry. Am J Med Genet A. 2020;182:1152-66.

122. Golzio C, Katsanis N. Genetic architecture of reciprocal CNVs. Curr Opin Genet Dev. 2013;23:240-8

\section{ACKNOWLEDGEMENTS}

This study was supported by NIH R01 MH110701 (J.G.M. and G.J.B.), F32 MH124273 (R.H.P.), and the Emory University School of Medicine. The GTEx data (release version 6) used for the analyses described in this manuscript were downloaded from the GTEx portal on 01/08/2019 (http://www.gtexportal.org/home/datasets/, file name: "GTEx_Analysis_v6_RNA-seq_RNA-SeQCv1.1.8_gene_rpkm.gct.gz"); dbGaP accession number: phs000424.v6.p1. The Genotype-Tissue Expression (GTEx) Project was supported by the Common Fund of the Office of the Director of the National Institutes of Health, and by $\mathrm{NCl}$, NHGRI, NHLBI, NIDA, NIMH, and NINDS. The BrainSpan Developmental
Transcriptome dataset used to construct the test network was downloaded from the Allen Brain Atlas portal on 01/12/2019 (https://www.brainspan.org/static/download/, file name: "RNA-Seq Gencode v10 summarized to genes"); dbGaP accession number: phs000755.v2.p1. Figures 1, 2, and 5 were created with illustrations from BioRender. com. The authors gratefully acknowledge the contributions of the members of the Emory 3q29 Project: Katrina Aberizk, Hallie Averbach, T. Lindsey Burrell, Shanthi Cambala, Grace Carlock, Tamara Caspary, Joseph F. Cubells, David Cutler, Paul A. Dawson, Michael P. Epstein, Roberto Espana, Michael J. Gambello, Katrina Goines, Ryan Guest, Henry R. Johnston, Cheryl Klaiman, Sookyong Koh, Elizabeth J. Leslie, Longchuan Li, Bryan Mak, Tamika Malone, Michael Mortillo, Trenell Mosley, Melissa M. Murphy, Derek Novacek, Rebecca M. Pollak, Rossana Sanchez, Celine A. Saulnier, Jason Schroeder, Sarah Shultz, Nikisha Sisodiya, Steven Sloan, Stephen T. Warren, David Weinshenker, Zhexing Wen, Stormi White, and Michael E. Zwick.

\section{CONFLICT OF INTEREST}

The authors declare no competing interests.

\section{ADDITIONAL INFORMATION}

Supplementary information The online version contains supplementary material available at https://doi.org/10.1038/s41398-021-01435-2.

Correspondence and requests for materials should be addressed to J.G.M.

Reprints and permission information is available at http://www.nature.com/ reprints

Publisher's note Springer Nature remains neutral with regard to jurisdictional claims in published maps and institutional affiliations.

(i) Open Access This article is licensed under a Creative Commons Attribution 4.0 International License, which permits use, sharing, adaptation, distribution and reproduction in any medium or format, as long as you give appropriate credit to the original author(s) and the source, provide a link to the Creative Commons license, and indicate if changes were made. The images or other third party material in this article are included in the article's Creative Commons license, unless indicated otherwise in a credit line to the material. If material is not included in the article's Creative Commons license and your intended use is not permitted by statutory regulation or exceeds the permitted use, you will need to obtain permission directly from the copyright holder. To view a copy of this license, visit http://creativecommons. org/licenses/by/4.0/.

(c) The Author(s) 2021

\section{THE EMORY 3Q29 PROJECT}

Katrina Aberizk ${ }^{2}$, Hallie Averbach ${ }^{1}$, Emily Black ${ }^{1}$, T. Lindsey Burrell ${ }^{6}$, Shanthi Cambala ${ }^{1}$, Grace Carlock ${ }^{1}$, Tamara Caspary ${ }^{1}$, Joseph F. Cubells ${ }^{1}$, David Cutler ${ }^{1}$, Paul A. Dawson ${ }^{6}$, Michael T. Epstein ${ }^{1}$, Roberto Espana ${ }^{2}$, Michael J. Gambello ${ }^{1}$, Katrina Goines ${ }^{2}$, Ryan M. Guest ${ }^{2}$, Henry R. Johnston ${ }^{1}$, Cheryl Klaiman ${ }^{2}$, Sookyong Koh ${ }^{2}$, Elizabeth J. Leslie ${ }^{1}$, Longchuan Li ${ }^{2}$, Bryan Mak ${ }^{1}$, Tamika Malone ${ }^{1}$, Trenell Mosley ${ }^{1}$, Melissa M. Murphy ${ }^{1}$, Ava Papetti ${ }^{1}$, Rebecca M. Pollak ${ }^{1}$, Rossana Sanchez Russo ${ }^{1}$, Celine A. Saulnier ${ }^{2}$, Sarah Shultz ${ }^{2}$, Nikisha Sisodoya ${ }^{1}$, Steven Sloan ${ }^{1}$, Stephen T. Warren ${ }^{1}$, David Weinshenker ${ }^{1}$, Zhexing Wen ${ }^{3}$, Stormi Pulver White ${ }^{2}$ and Mike Zwick ${ }^{1}$ 